\title{
Multi-colour photometry of nearby dwarf galaxies ${ }^{\star, \star \star}$
}

\author{
L. Makarova \\ Special Astrophysical Observatory, Russian Academy of Sciences, N. Arkhyz, KChR, 357147, Russia
}

Received June 25; accepted August 5, 1999

\begin{abstract}
Observations of 39 nearby, mostly dwarf galaxies are presented. The observations were carried out at the 1.2-m telescope of Observatoire de Haute-Provence (France) with $B, V$ and $I$ Cousins filters. Based on surface and integrated photometry of the obtained images we derived total $B, V$ and $I$ magnitudes and integrated $B-V, V-I$ colours as well as radii and magnitudes at the $25^{\mathrm{m}}$ isophotal level.

Azimuthally averaged surface brightness profiles were derived for 33 galaxies in each photometric band. Most of the profiles can be well fitted by an exponential intensity law of brightness distribution. The best-fitting exponential parameters are also given for the galaxies.
\end{abstract}

Key words: galaxies: fundamental parameters - galaxies: photometry - galaxies: irregular — galaxies: structure

\section{Introduction}

Nearby dwarf galaxies are well recognized now as central objects under study for many areas of extragalactic astronomy, such as star formation, galaxy evolution and structure (Stetson et al. 1998; Greggio et al. 1998). Many nearby galaxies are also well resolved into individual stars, which allows one to estimate photometric galaxy distances (see, for example, Karachentsev et al. 1994; Makarova \& Karachentsev 1998). Nevertheless, the main photometric parameters, such as total magnitudes and colours, remain poorly known for a number of nearby dwarf galaxies. A study of these galaxies not only in visual bands ( $B$ and $V$ Johnson-Cousins filters), but also in the near infrared ( $I$

\footnotetext{
* Based on observations made at Observatoire de Haute Provence (CNRS), France.

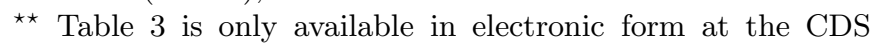
via anonymous ftp to cdsarc.u-strasbg.fr (130.79.128.5) or via http://cdsweb.u-strasbg.fr/Abstract.html
}

Cousins filter), where extinction is less, can make it possible to investigate in detail the galaxy morphology and structure (Heraudeau \& Simien 1996).

A sample of nearby, mostly dwarf irregular galaxies with significant uncertainty of total magnitude $B_{\mathrm{T}}$ was selected from the Principal Galaxy Catalogue (Paturel et al. 1996). CCD observations of galaxies from this list are presented in this paper. The observations were carried out at the 1.2-m telescope of Observatoire de HauteProvence (OHP), France with $B, V$ and $I$ Cousins filters (see Sect. 3). Total magnitudes of observed galaxies were derived in each photometric band and azimuthally averaged surface brightness profiles were also constructed (see Sect. 5). The profiles were fitted by an exponential intensity law or by a combination of two such functions. The best-fitting parameters are given in Sect. 6 .

\section{The sample}

The galaxies for our sample were extracted mostly from the PGC catalogue (Paturel et al. 1996). The northern $\left(\delta>0^{\circ}\right)$ objects with radial velocities $<500 \mathrm{~km} \mathrm{~s}^{-1}$ and with an uncertainty of total magnitude $B_{\mathrm{T}}>0.2^{\mathrm{m}}$ were included in the list. Some objects from the list of nearby galaxies by Karachentsev (1994) were also added to the sample. General parameters of 61 chosen galaxies are given in Table 1. The columns of the table are:

Column 1: NGC, IC or UCG name of the galaxy.

Column 2: PGC name.

Columns 3 and 4: 1950 coordinates.

Column 5: galaxy type according to RC3 system, as given in PGC catalogue.

Column 6: angular diameter at the $25^{\mathrm{m}}$ isophotal level (in arcmin) from PGC.

Column \%: total magnitude $B_{\mathrm{T}}$ according to PGC.

Columns 8, 9 and 10: values of galactic absorption (in mag) in $B, V$ and $I$ filters, respectively, taken from IRAS/DIRBE maps (Schlegel et al. 1998).

Column 11: radial velocity in $\mathrm{km} \mathrm{s}^{-1}$. 
Table 1. General parameters of the sample

\begin{tabular}{|c|c|c|c|c|c|c|c|c|c|c|c|}
\hline Ident. & PGC id. & $\alpha(1950)$ & $\delta(1950)$ & Type & $\begin{array}{c}D_{25} \\
(\operatorname{arcmin})\end{array}$ & $B_{\mathrm{T}}$ & $A_{B}$ & $A_{V}$ & $A_{I}$ & $\begin{array}{c}V_{\odot} \\
\left(\mathrm{km} \mathrm{s}^{-1}\right)\end{array}$ & $\begin{array}{c}\text { Dist. } \\
(\mathrm{Mpc})\end{array}$ \\
\hline IC 10 & PGC 0001305 & $00^{\mathrm{h}} 17^{\mathrm{m}} 41 .^{\mathrm{s}} 5$ & $59^{\circ} 00^{\prime} 52^{\prime \prime}$ & 9.8 & 6.8 & 12.20 & 4.01 & 3.08 & 1.80 & -345 & 0.8 \\
\hline Cas 1 & LEDA0100169 & 020205.0 & 684618 & & & & 4.44 & 3.42 & 2.00 & 35 & 2.8 \\
\hline UGCA 39 & PGC 0010217 & 023807.9 & 592324 & 3.9 & 3.3 & & 5.73 & 4.40 & 2.58 & -2 & 2.7 \\
\hline Dwingeloo 2 & LEDA0101304 & 025021.7 & 584807 & & & & 5.11 & 3.93 & 2.30 & 93 & 4.5 \\
\hline Dwingeloo 1 & LEDA0100170 & 025306.4 & 584238 & & & & 5.94 & 4.57 & 2.67 & 110 & 4.8 \\
\hline NGC 1156 & PGC 0011329 & 025646.8 & 250221 & 9.8 & 3.3 & 12.04 & 1.06 & 0.82 & 0.48 & 380 & 7.8 \\
\hline UGC 2684 & PGC 0012514 & 031742.0 & 170800 & 9.9 & 1.8 & & 0.58 & 0.45 & 0.26 & 349 & 6.5 \\
\hline UGC 2716 & PGC 0012719 & 032117.2 & 173442 & 7.9 & 1.6 & 15.13 & 0.61 & 0.47 & 0.27 & 379 & 6.7 \\
\hline UGC 2773 & PGC 0013115 & 032835.3 & 473728 & & 1.2 & 15.04 & 2.37 & 1.82 & 1.06 & 242 & 5.6 \\
\hline UGCA 86 & PGC 0014241 & 035500.0 & 665900 & 10.0 & 0.9 & & 4.22 & 3.24 & 1.90 & 68 & 2.6 \\
\hline NGC 1569 & PGC 0015345 & 042605.8 & 644418 & 9.8 & 3.6 & 11.70 & 3.40 & 2.61 & 1.53 & -86 & 1.7 \\
\hline UGCA 92 & PGC 0015439 & 042724.0 & 633000 & 10.0 & 2.0 & & 3.58 & 2.75 & 1.61 & -99 & 1.8 \\
\hline UGCA 105 & PGC 0016957 & 050936.0 & 623100 & 10.0 & 5.5 & 15.25 & 1.38 & 1.06 & 0.62 & 111 & 3.2 \\
\hline KK 49 & PGC 0017603 & 053900.0 & 064000 & & & 15.36 & 2.90 & 2.23 & 1.30 & 441 & 5.4 \\
\hline Orion & PGC 0017716 & 054222.0 & 050230 & & & & 2.84 & 2.18 & 1.28 & 365 & 6.4 \\
\hline UGC 3676 & PGC 0019162 & 062711.9 & 332013 & 9.9 & 1.0 & 15.67 & 1.00 & 0.76 & 0.45 & 469 & 7.0 \\
\hline UGC 3600 & PGC 0019871 & 065212.0 & 390918 & 10.0 & 1.1 & & 0.40 & 0.31 & 0.18 & 403 & 7.3 \\
\hline UGC 3698 & PGC 0020264 & 070541.4 & 442742 & 10.0 & 1.0 & 15.43 & 0.45 & 0.34 & 0.20 & 423 & 7.2 \\
\hline NGC 2337 & PGC 0020298 & 070637.1 & 443220 & 9.9 & 2.0 & 13.05 & 0.40 & 0.31 & 0.18 & 434 & 7.9 \\
\hline UGC 3755 & PGC 0020445 & 071106.3 & 103618 & 9.9 & 1.6 & 14.64 & 0.38 & 0.29 & 0.17 & 323 & 4.1 \\
\hline \multirow[t]{2}{*}{ UGC 3817} & PGC 0020852 & 071906.0 & 451200 & 10.0 & 1.8 & & 0.41 & 0.32 & 0.18 & 438 & 8.6 \\
\hline & LEDA0093088 & 072312.0 & 691700 & 8.0 & 1.1 & & 0.17 & 0.13 & 0.08 & 135 & \\
\hline NGC 2366 & PGC 0021102 & 072336.5 & 691900 & 9.7 & 7.3 & 11.43 & 0.16 & 0.12 & 0.07 & 99 & 3.4 \\
\hline UGC 3974 & PGC 0021600 & 073902.7 & 165517 & 9.8 & 3.8 & 13.71 & 0.15 & 0.12 & 0.07 & 270 & 4.3 \\
\hline K 52 & PGC 0023521 & 081843.0 & 711125 & 10.0 & 1.3 & 16.5 & 0.11 & 0.08 & 0.05 & 113 & 3.0 \\
\hline UGC 4459 & PGC 0024050 & 082933.0 & 662101 & 9.8 & 1.6 & 14.5 & 0.15 & 0.12 & 0.07 & 18 & 3.1 \\
\hline UGC 4483 & PGC 0024213 & 083207.0 & 695716 & 9.9 & 1.1 & 15.51 & 0.14 & 0.11 & 0.06 & 156 & 3.6 \\
\hline F464-v3 & LEDA 086668 & 090003.0 & 201619 & 10.0 & 0.9 & 15.9 & 0.13 & 0.10 & 0.06 & 481 & 5.2 \\
\hline UGC 5086 & PGC 0027115 & 092959.1 & 214112 & 8.4 & 0.9 & 15.73 & 0.14 & 0.11 & 0.06 & 498 & 7.4 \\
\hline Holmberg I & PGC 0027605 & 093600.0 & 712447 & 9.8 & 3.6 & 13.20 & 0.18 & 0.14 & 0.08 & 136 & 6.7 \\
\hline UGC 5288 & PGC 0028378 & 094838.5 & 080343 & 7.9 & 0.8 & 14.22 & 0.15 & 0.12 & 0.07 & 555 & 6.8 \\
\hline BK3N & PGC 0028529 & 094942.0 & 691218 & 10.0 & 0.5 & 18.6 & 0.38 & 0.29 & 0.17 & -40 & 2.8 \\
\hline Holmberg IX & PGC 0028757 & 095328.0 & 691653 & 9.8 & 2.6 & 14.41 & 0.34 & 0.26 & 0.16 & 47 & 3.4 \\
\hline UGC 5423 & PGC 0029284 & 100125.3 & 703627 & 9.9 & 1.0 & 15.52 & 0.36 & 0.28 & 0.16 & 342 & 5.3 \\
\hline UGC 5692 & PGC 0030997 & 102648.0 & 705233 & 8.6 & 3.4 & 13.49 & 0.20 & 0.15 & 0.09 & 180 & 4.5 \\
\hline UGC 5918 & PGC 0032405 & 104617.0 & 654740 & 9.8 & 2.4 & 15.13 & 0.05 & 0.04 & 0.02 & 338 & 7.4 \\
\hline K 73 & PGC 0032667 & 104928.2 & 694842 & 10.0 & 0.6 & 18.2 & 0.09 & 0.07 & 0.04 & 115 & 4.0 \\
\hline UGC 6817 & PGC 0037050 & 114816.8 & 390931 & 9.9 & 4.2 & 13.44 & 0.11 & 0.09 & 0.05 & 245 & 3.9 \\
\hline NGC 4068 & PGC 0038148 & 120129.7 & 525201 & 9.8 & 3.2 & 13.21 & 0.08 & 0.06 & 0.04 & 210 & 5.2 \\
\hline NGC 4144 & PGC 0038688 & 120728.3 & 464407 & 6.0 & 6.2 & 12.01 & 0.07 & 0.05 & 0.03 & 267 & 9.8 \\
\hline NGC 4163 & PGC 0038881 & 120937.6 & 362651 & 9.8 & 1.7 & 14.48 & 0.09 & 0.07 & 0.04 & 163 & 3.6 \\
\hline KK 127 & PGC 0038998 & 121051.1 & 301159 & & 1.0 & 15.61 & 0.08 & 0.06 & 0.04 & 152 & 16.0 \\
\hline UGC 7298 & PGC 0039316 & 121400.0 & 523200 & 9.8 & 1.2 & & 0.11 & 0.08 & 0.05 & 170 & 8.6 \\
\hline UGC 7321 & PGC 0039432 & 121502.3 & 224901 & 6.5 & 5.6 & 14.11 & 0.11 & 0.08 & 0.05 & 409 & 4.9 \\
\hline
\end{tabular}


Table 1. continued

\begin{tabular}{lcccccccccccc}
\hline Ident. & PGC id. & $\alpha(1950)$ & $\delta(1950)$ & Type & $\begin{array}{c}D_{25} \\
(\operatorname{arcmin})\end{array}$ & $B_{\mathrm{T}}$ & $A_{B}$ & $A_{V}$ & $A_{I}$ & $\begin{array}{c}V_{\odot} \\
\left(\mathrm{km} \mathrm{s}^{-1}\right)\end{array}$ & $\begin{array}{c}\text { Dist. } \\
(\mathrm{Mpc})\end{array}$ \\
\hline IC 3308 & PGC 0040495 & 122248.0 & 265931 & 7.3 & 1.4 & 15.17 & 0.09 & 0.07 & 0.04 & 315 & 3.9 \\
UGC 7577 & PGC 0040904 & 122515.4 & 434613 & 9.7 & 4.2 & 12.87 & 0.08 & 0.06 & 0.04 & 195 & 4.8 \\
NGC 4449 & PGC 0040973 & 122545.2 & 442215 & 9.7 & 6.2 & 9.83 & 0.07 & 0.05 & 0.03 & 202 & 4.5 \\
UGC 7698 & PGC 0041636 & 123026.2 & 314902 & 9.9 & 6.5 & 13.06 & 0.06 & 0.05 & 0.03 & 332 & 6.1 \\
IC 3687 & PGC 0042656 & 123950.9 & 384633 & 9.8 & 3.5 & 13.72 & 0.08 & 0.06 & 0.04 & 358 & 3.0 \\
IC 4182 & PGC 0045314 & 130330.0 & 375223 & 8.7 & 6.0 & 12.41 & 0.06 & 0.05 & 0.03 & 315 & 5.0 \\
UGC 8201 & PGC 0045372 & 130439.4 & 675816 & 9.9 & 3.4 & 12.85 & 0.11 & 0.08 & 0.05 & 34 & 4.9 \\
UGC 8308 & PGC 0045939 & 131111.9 & 463457 & 9.8 & 1.1 & 14.91 & 0.05 & 0.04 & 0.02 & 164 & 3.7 \\
UGC 8320 & PGC 0046039 & 131216.6 & 461101 & 9.9 & 3.6 & 12.73 & 0.06 & 0.04 & 0.02 & 194 & 4.0 \\
UGC 8331 & PGC 0046127 & 131320.3 & 474537 & 9.8 & 2.5 & 14.59 & 0.03 & 0.02 & 0.02 & 259 & 8.2 \\
UGC 8508 & PGC 0047495 & 132846.8 & 551003 & 9.8 & 1.7 & 14.64 & 0.06 & 0.04 & 0.03 & 62 & 3.7 \\
NGC 5229 & PGC 0047788 & 133158.5 & 481016 & 6.6 & 3.3 & 14.51 & 0.06 & 0.05 & 0.03 & 364 & 5.1 \\
Holmberg IV & PGC 0049448 & 135255.2 & 540858 & 9.7 & 4.2 & 13.59 & 0.06 & 0.05 & 0.03 & 142 & 8.3 \\
NGC 5474 & PGC 0050216 & 140315.3 & 535405 & 6.0 & 5.0 & 11.23 & 0.05 & 0.04 & 0.02 & 276 & 7.2 \\
NGC 6503 & PGC 0060921 & 174958.7 & 700926 & 5.9 & 7.1 & 10.74 & 0.15 & 0.11 & 0.06 & 44 & 5.2 \\
NGC 6946 & PGC 0065001 203348.8 & 595850 & 5.9 & 11.2 & 9.58 & 1.48 & 1.14 & 0.66 & 52 & 6.0 \\
Pegasus & PGC 0071538 232602.8 & 142816 & 9.8 & 5.1 & 12.82 & 0.27 & 0.20 & 0.12 & -183 & 0.8 \\
\hline
\end{tabular}

Column 12: galaxy distance in Mpc taken from Karachentsev (1994), recently revised by D. Makarov and I. Karachentsev (private communications).

The majority of the galaxies from our list have small diameters $(1-4$ arcmin $)$ and type around $9.5-10$. The distribution of their total magnitudes $B_{\mathrm{T}}$ shows a maximum near 13 and 15 magnitudes. Therefore, most of the sample galaxies are dwarf irregular objects.

\section{Observations}

39 of the galaxies from our sample were observed on April, 1995 at the 1.2-m telescope of Observatoire de Haute-Provence (OHP), France. A CCD camera No. 2 Tektronix $1024 \times 1024$ pix provided a field of view of $11.8^{\prime} \times 11.8^{\prime}$ with a resolution of $0.69^{\prime \prime}$ per pixel. $B, V$ and $I$ Cousins filters were used (see the equipment description on http://www.obs-hp.fr). The list of the galaxy exposures is presented in Table 2, where the columns are:

Column 1: galaxy name.

Column 2: date of the observation.

Columns 3, 4 and 5: exposure time (in s) in $B, V$ or $I$ filter, respectively.

Some bias and dark exposures were made every night. Flat fields (twilight sky) were also exposed every evening and every morning.

\section{Data reduction}

\subsection{Initial processing}

Reduction of the obtained images was done with the MIDAS package developed by ESO. Initial processing of the data contained usual steps such as bias and dark frame subtraction and flat-fielding. Cosmic rays were then removed from each galaxy image with the FILTER/COSMIC procedure. The galaxy frames of the same band were summed, if several exposures were made. Images of the observed galaxies in $B$ band are presented in Fig. 1.

\subsection{Sky background determination}

Background stars were removed from the frames by fitting a second-degree surface in circular pixel-area. The sky background was then approximated by a tilted plane, created from a 2-dimension polynomial, using the leastsquares method (FIT/FLAT_SKY). The accuracy of the sky background determination is about $0.3 \%$ of the original sky level. A typical value of the sky surface brightness is near $22^{\mathrm{m}} / \mathrm{sq} \mathrm{s}$ in $B$ band. Therefore, the mean error of sky determination must not exceed 0.07 mag.

The center coordinates for about $1 / 3$ of all the observed galaxies were determined by ellipse fitting of outer parts of the galaxy (FIT/ELL3). Most of other galaxy centers were determined interactively, because a significant part of the sample consists of the galaxies of irregular morphology. For some galaxies with a star-like center the coordinates of the center were determined using first moment of the intensity pixel values.

\subsection{Photometric calibration}

For the calibration, the photometric standard stars from Landolt's (1992) list were observed during the observational run after every two galaxies. Due to extinction 


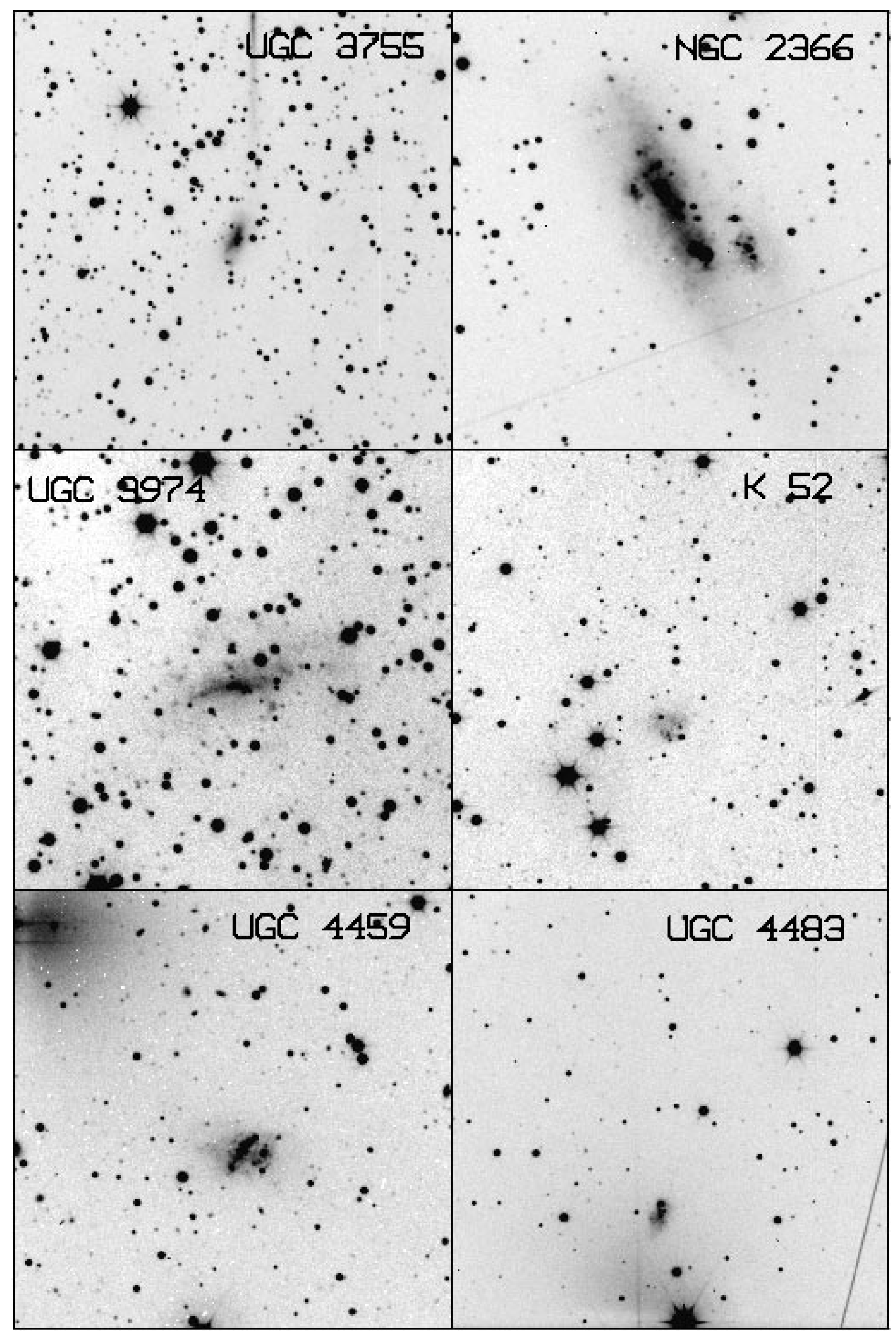

Fig. 1. The images of the sample galaxies in $B$ band, which were obtained at the 1.2-m telescope of Observatoire de HauteProvence (France). The field size of the images is $11.8^{\prime} \times 11.8^{\prime}$. For all the images North is top and East is left 


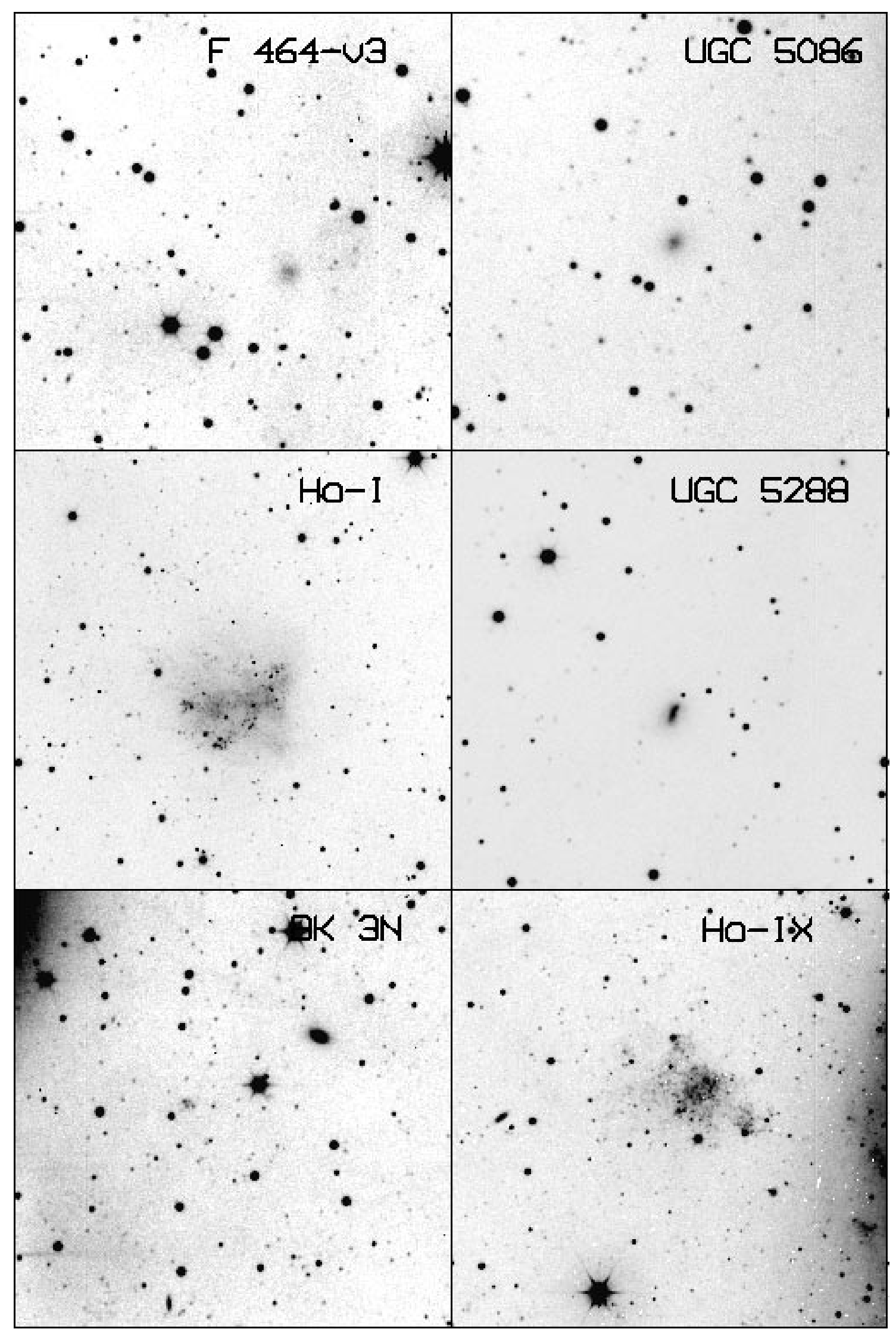

Fig. 1. continued 


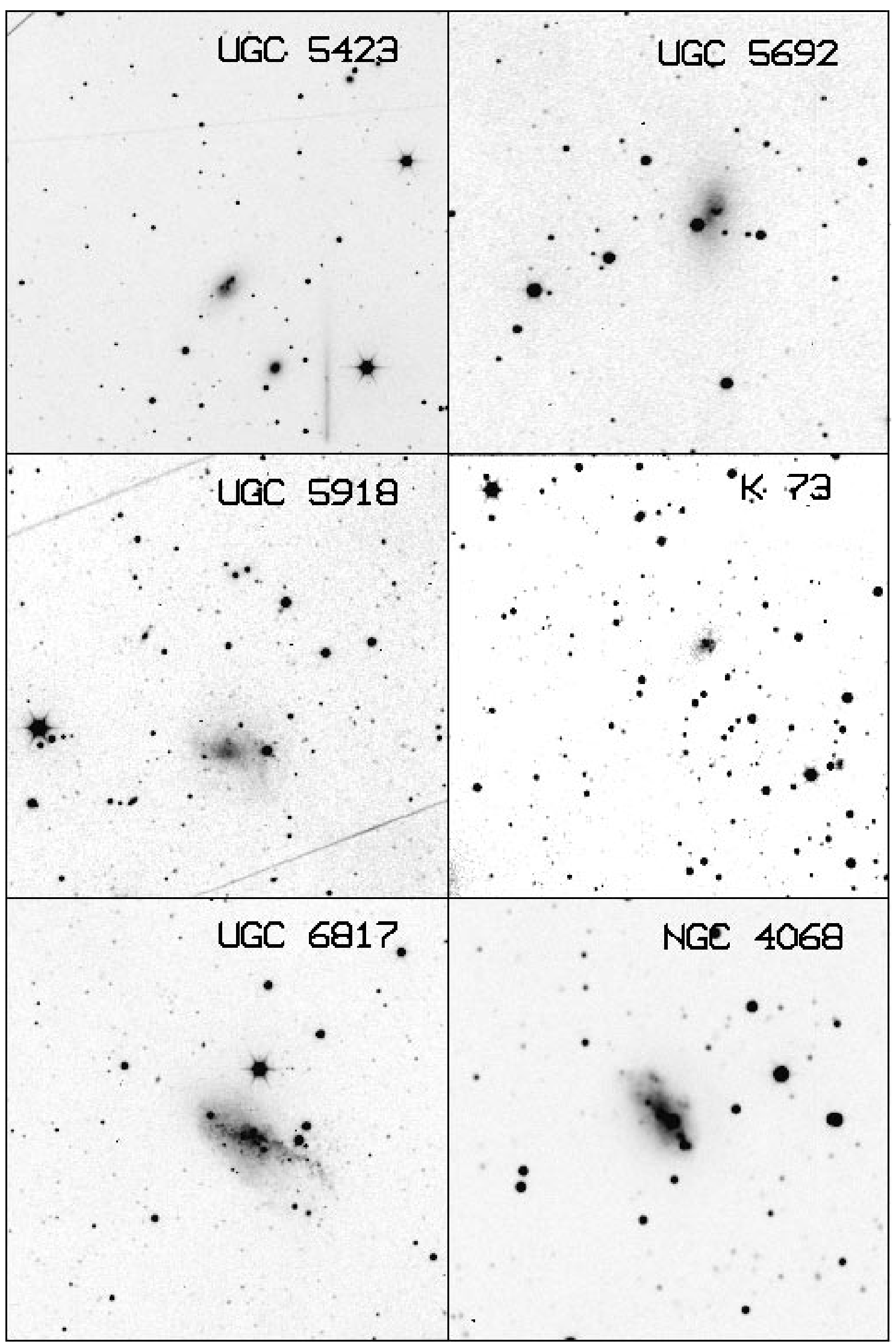

Fig. 1. continued 


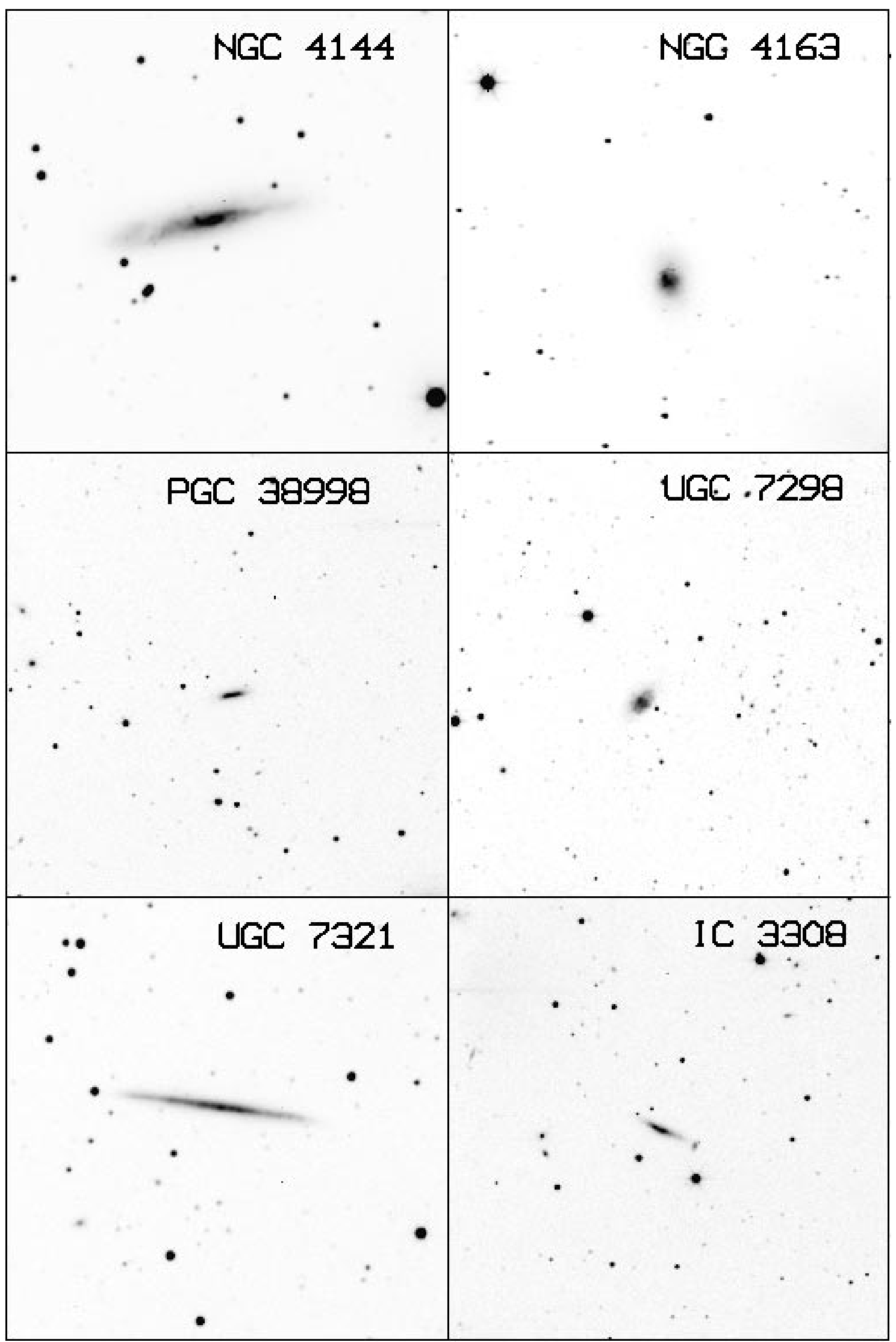

Fig. 1. continued 


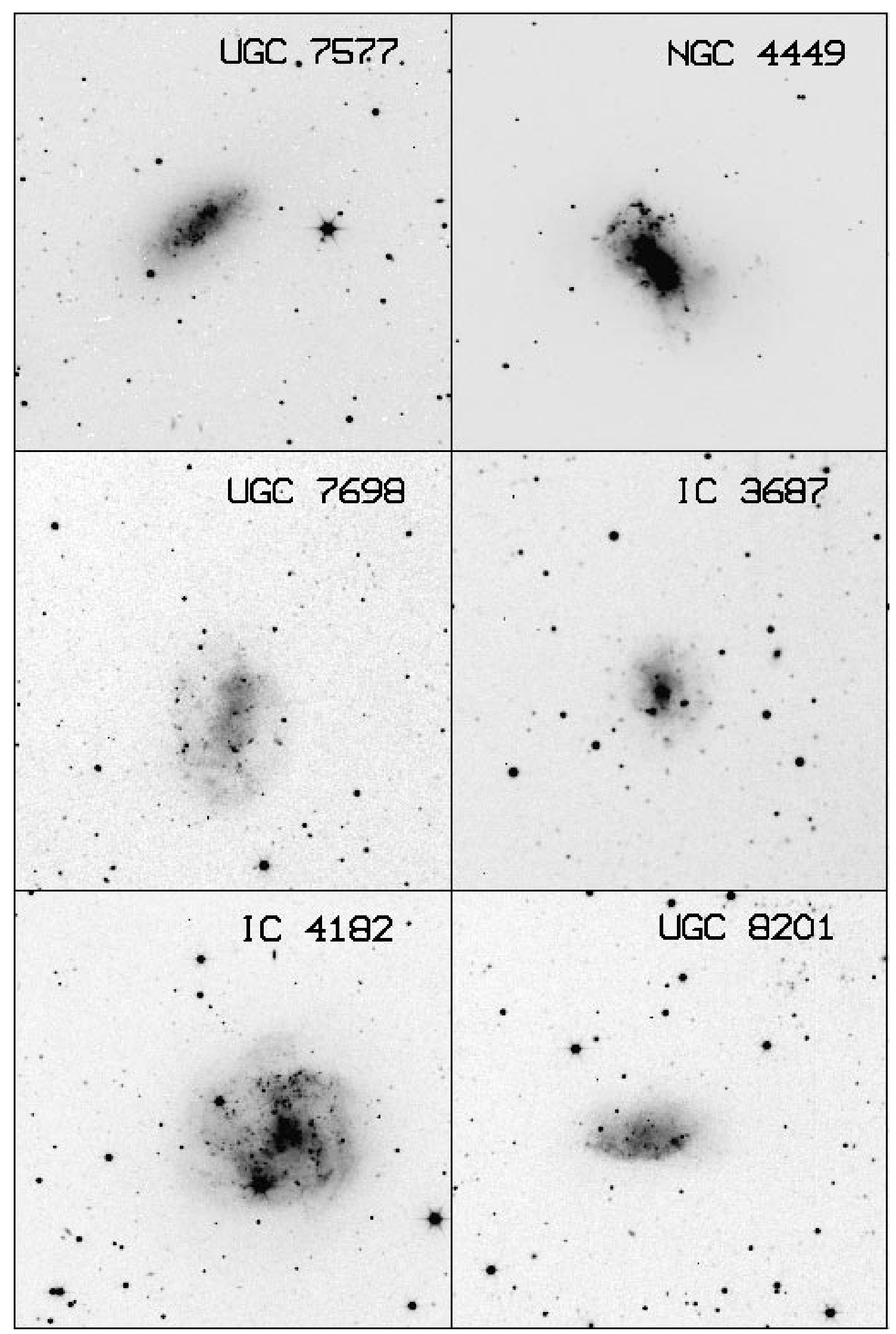

Fig. 1. continued 


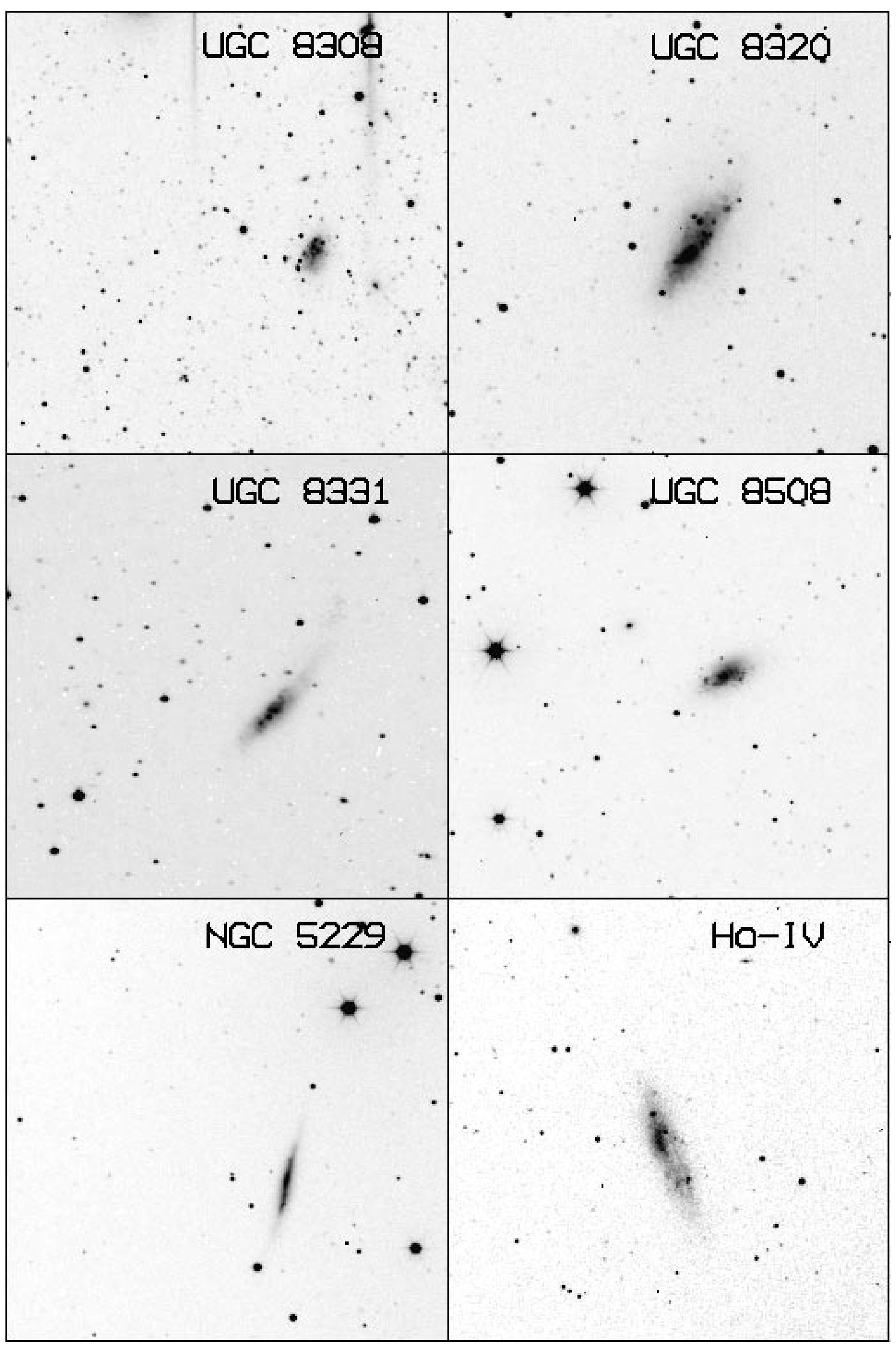

Fig. 1. continued 


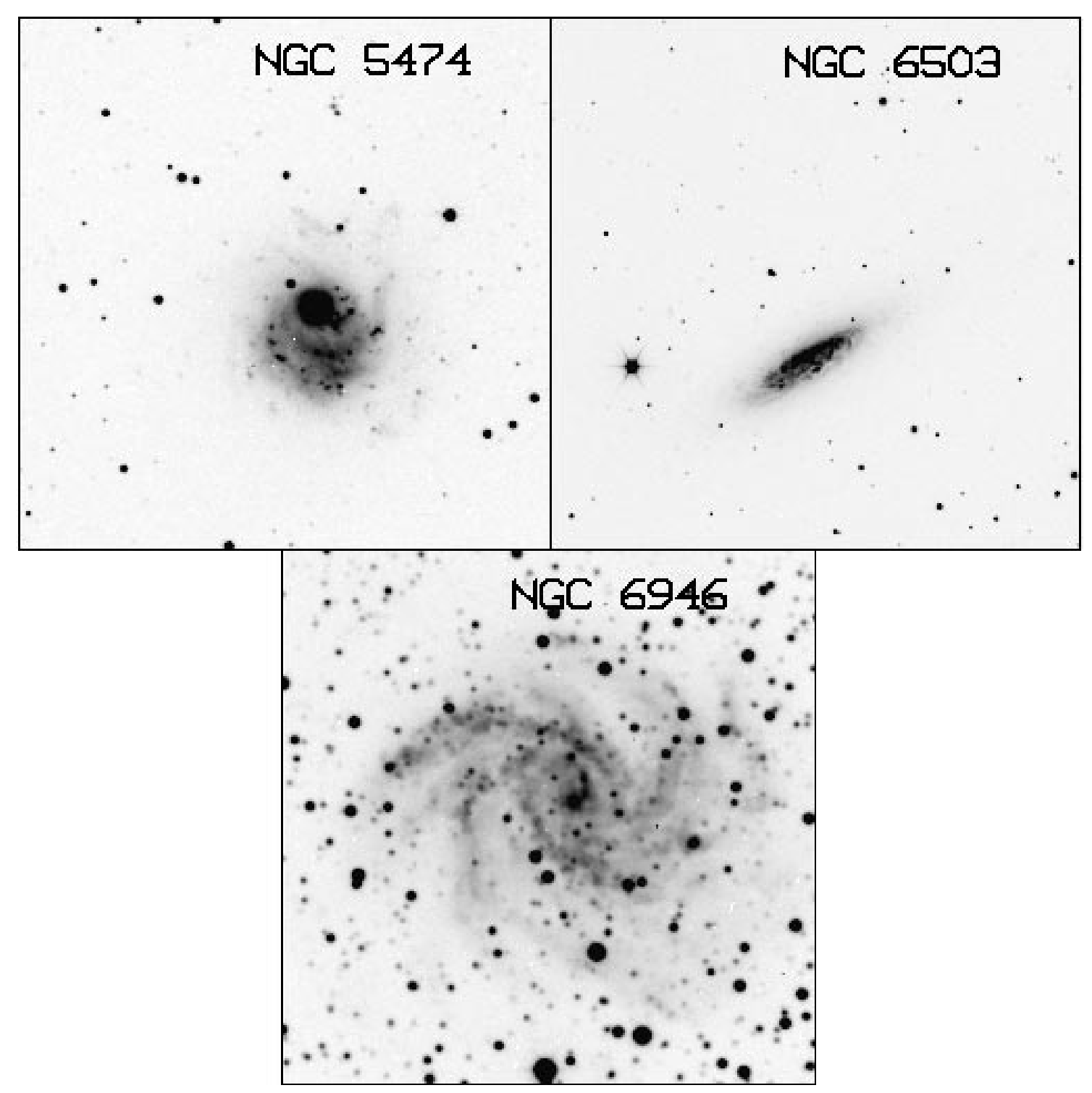

Fig. 1. continued

present on some nights, a number of the galaxies were exposed once more with short exposures under better photometric conditions (see Table 2). Deeper images were then calibrated using these short-exposure data.

Zero-point errors of the transformation to the standard Johnson-Cousins system are estimated to be about $0.15^{\mathrm{m}}$ in each filter.

\section{Results}

\subsection{Total magnitudes and surface brightness profiles}

For estimation of total galaxy magnitudes in each band, integrated photometry was performed in increasing circular apertures from a prechosen center (see above) to faint outskirts of the galaxies. Aperture photometry of the observed galaxies is presented in Table 3 , which is available only in electronic form in the CDS. The total magnitude was estimated as asymptotic value of the obtained radial growth curve. The asymptotic extrapolation was done by eye. The errors of the total magnitude determination are $0.06^{\mathrm{m}}$ in $B$ band, $0.08^{\mathrm{m}}$ in $V$ and $0.10^{\mathrm{m}}$ in $I$. The results of our measurements are presented in Table 4 , where the columns represent:

Column 1: galaxy name.

Columns 2, 3 and 4: total magnitudes in $B, V$ and $I$ band, respectively (not corrected for galactic absorption).

Columns 5 and 6: total colour $B-V$ and $V-I$, respectively (not corrected for galactic absorption).

Column \%: radius (arcsec) at the surface brightness level of $25^{\mathrm{m}} / \mathrm{sq} \mathrm{s}$ in $B$ band.

Column 8: total B-magnitude at this level (not corrected for galactic absorption). 
Table 2. Observational log

\begin{tabular}{|c|c|c|c|c|}
\hline Ident. & Date & $B$ expos.(sec) & $V$ expos.(sec) & $I$ expos.(sec) \\
\hline UGC 3755 & 11.04 .1997 & $2 \times 400$ & $2 \times 400$ & $2 \times 500$ \\
\hline NGC 2366 & 05.04 .1997 & 600 & 500 & 600 \\
\hline UGC 3974 & 11.04.1997 & $2 \times 500$ & $2 \times 500$ & $2 \times 600$ \\
\hline K 52 & 10.04.1997 & $2 \times 600$ & $2 \times 600$ & $2 \times 900$ \\
\hline UGC 4459 & 05.04 .1997 & $2 \times 600$ & $2 \times 500$ & $2 \times 600$ \\
\hline UGC 4483 & 09.04.1997 & $2 \times 400$ & $2 \times 400$ & $2 \times 600$ \\
\hline F464-v3 & 12.04 .1997 & $3 \times 600$ & $3 \times 600$ & $3 \times 900$ \\
\hline UGC 5086 & 11.04 .1997 & $2 \times 400$ & $2 \times 400$ & $2 \times 600$ \\
\hline Holmberg I & 02.04.1997 & $600+1200$ & 1000 & 800 \\
\hline UGC 5288 & 12.04 .1997 & $2 \times 400$ & $2 \times 400$ & $2 \times 600$ \\
\hline BK3N & 12.04 .1997 & $3 \times 600$ & $3 \times 600$ & $3 \times 900$ \\
\hline Holmberg IX & 06.04 .1997 & $2 \times 800$ & $2 \times 800$ & $2 \times 1000$ \\
\hline UGC 5423 & 07.04 .1997 & $2 \times 600$ & $2 \times 600$ & $2 \times 800$ \\
\hline \multirow[t]{2}{*}{ UGC 5692} & 03.04.1997 & $3 \times 400$ & $2 \times 300$ & $2 \times 300$ \\
\hline & 09.04 .1997 & 400 & 60 & 60 \\
\hline UGC 5918 & 09.04.1997 & $3 \times 500$ & $3 \times 500$ & $3 \times 600$ \\
\hline K 73 & 12.04 .1997 & $2 \times 600$ & $2 \times 600$ & $2 \times 900$ \\
\hline UGC 6817 & 02.04.1997 & $2 \times 600$ & $2 \times 500$ & $2 \times 450$ \\
\hline \multirow[t]{2}{*}{ NGC 4068} & 04.04.1997 & 600 & $2 \times 300$ & $2 \times 300$ \\
\hline & 10.04 .1997 & 100 & 100 & 100 \\
\hline \multirow[t]{2}{*}{ NGC 4144} & 04.04 .1997 & $2 \times 300$ & 300 & 300 \\
\hline & 10.04.1997 & 100 & 100 & 100 \\
\hline NGC 4163 & 05.04 .1997 & 600 & 500 & 700 \\
\hline PGC 38998 & 09.04.1997 & $2 \times 300$ & $2 \times 300$ & $2 \times 500$ \\
\hline UGC 7298 & 07.04.1997 & $3 \times 600$ & $3 \times 600$ & $3 \times 800$ \\
\hline \multirow[t]{2}{*}{ UGC 7321} & 04.04 .1997 & 1000 & 800 & 600 \\
\hline & 10.04 .1997 & 100 & 100 & 100 \\
\hline IC 3308 & 09.04.1997 & $2 \times 300$ & $2 \times 300$ & $2 \times 500$ \\
\hline UGC 7577 & 06.04 .1997 & $2 \times 400$ & $2 \times 400$ & $2 \times 500$ \\
\hline NGC 4449 & 02.04.1997 & 100 & 100 & 100 \\
\hline UGC 7698 & 10.04 .1997 & 600 & 600 & $2 \times 900$ \\
\hline \multirow[t]{2}{*}{ IC 3687} & 04.04 .1997 & $2 \times 400$ & $2 \times 400$ & $2 \times 400$ \\
\hline & 10.04 .1997 & 100 & 100 & 100 \\
\hline IC 4182 & 02.04.1997 & 600 & 500 & $2 \times 300$ \\
\hline UGC 8201 & 10.04 .1997 & 400 & 400 & $2 \times 600$ \\
\hline UGC 8308 & 02.04.1997 & $2 \times 1200$ & 1000 & $1000+900$ \\
\hline \multirow[t]{2}{*}{ UGC 8320} & 04.04 .1997 & $2 \times 400$ & $2 \times 400$ & $2 \times 500$ \\
\hline & 10.04.1997 & 100 & 100 & 100 \\
\hline UGC 8331 & 05.04.1997 & $2 \times 500$ & $2 \times 500$ & $2 \times 600$ \\
\hline UGC 8508 & 06.04.1997 & $2 \times 400$ & $2 \times 400$ & $2 \times 500$ \\
\hline NGC 5229 & 07.04.1997 & 600 & 600 & 900 \\
\hline \multirow[t]{2}{*}{ Holmberg IV } & 08.04.1997 & $2 \times 600$ & $2 \times 600$ & $3 \times 800$ \\
\hline & 10.04.1997 & 100 & 100 & 100 \\
\hline NGC 5474 & 06.04.1997 & 500 & 500 & 600 \\
\hline NGC 6503 & 02.04.1997 & 100 & 100 & 120 \\
\hline NGC 6946 & 05.04 .1997 & $3 \times 100$ & 100 & 100 \\
\hline
\end{tabular}

Columns 9 and 10: same as 7 and 8, but for $V$ band. Columns 11 and 12: same as 7 and 8, but for $I$ band. Column 13: absolute $B$ magnitude corrected for galactic absorption.

The blanks in Cols. 7-12 are due to the lack of surface brightness profiles for these galaxies (see below). The magnitude values for the galaxy UGC 5086 are uncertain because of extinction and marked with a colon.
Obtaining of the galaxy growth curves allows us also to trace the integrated colour changes with radius. $B-V$ and $V-I$ total colours versus galaxy radius from center are given in Fig. 2 for 33 galaxies of the sample. The growth curves were not determined for the six dwarf galaxies (K 52, UGC 4483, F464-v3, UGC 5086, BK3N, K 73) because of significant measurement errors caused by the extremely low surface brightness of the object or by bright nearby background stars, which affects photometry 

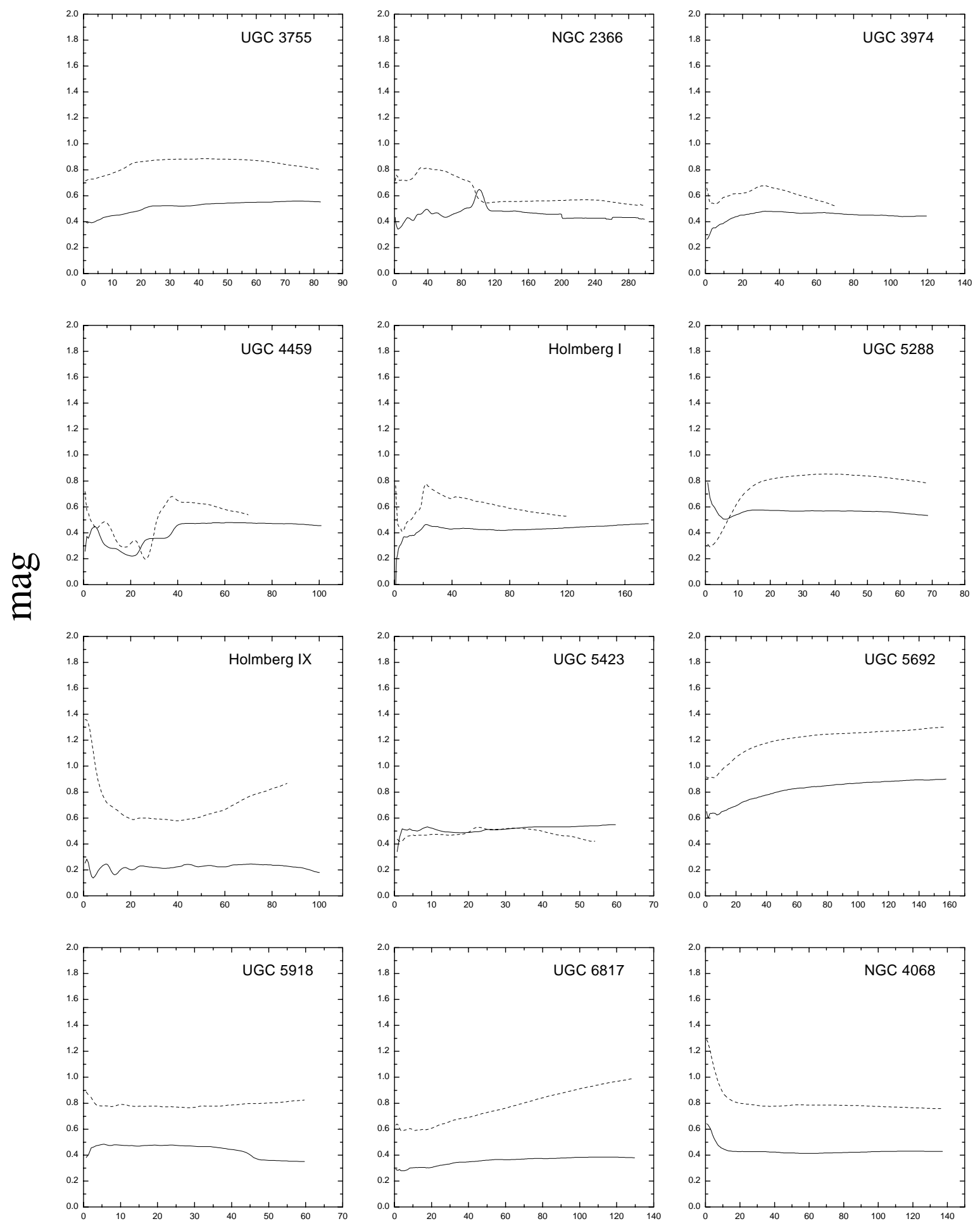

\section{Radius (arcsec)}

Fig. 2. The total $B-V$ colour (solid line) and the total $V-I$ colour (dashed line) with respect to the radius from the galaxy center $(\operatorname{arcsec})$ 

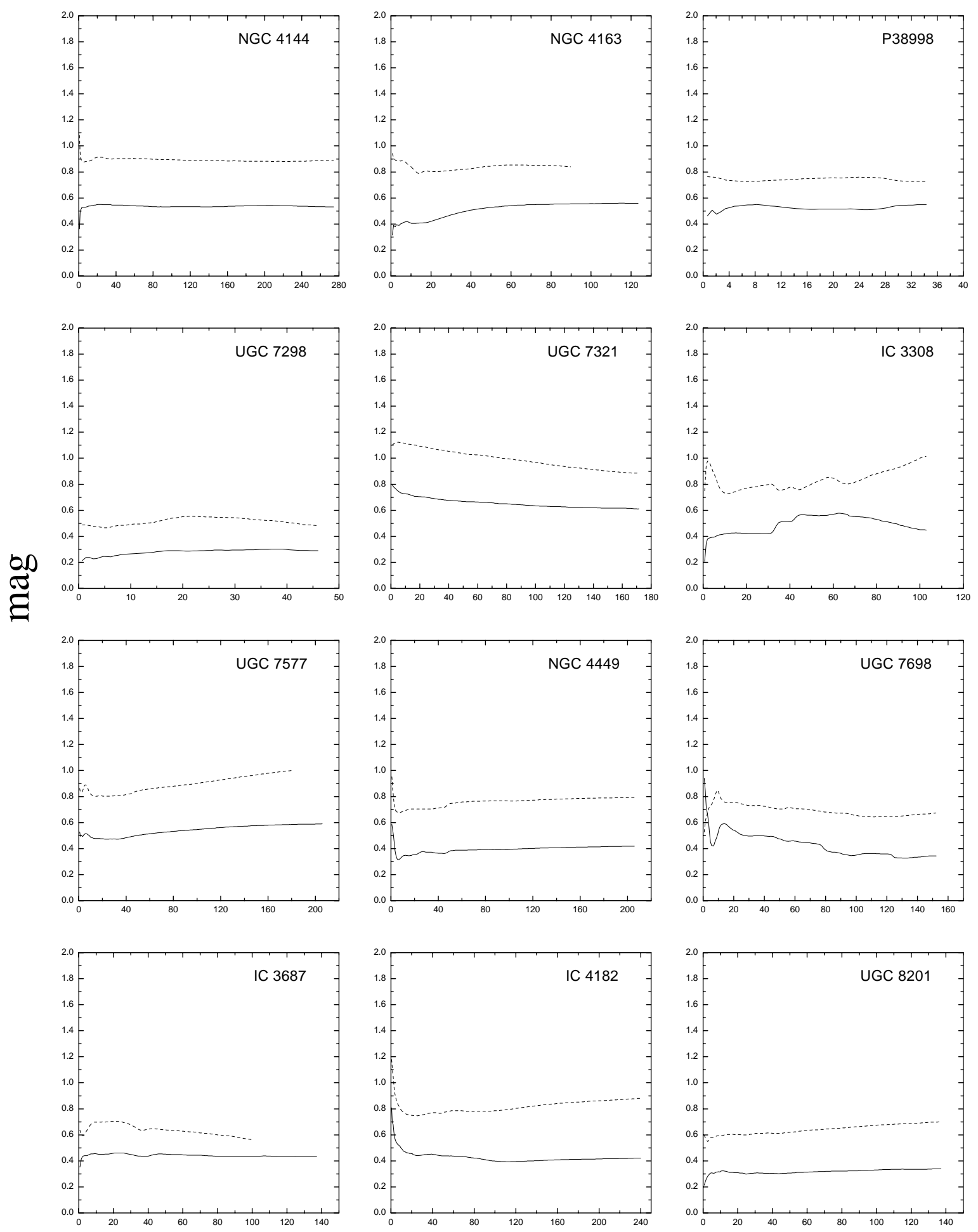

\section{Radius (arcsec)}

Fig. 2. continued 

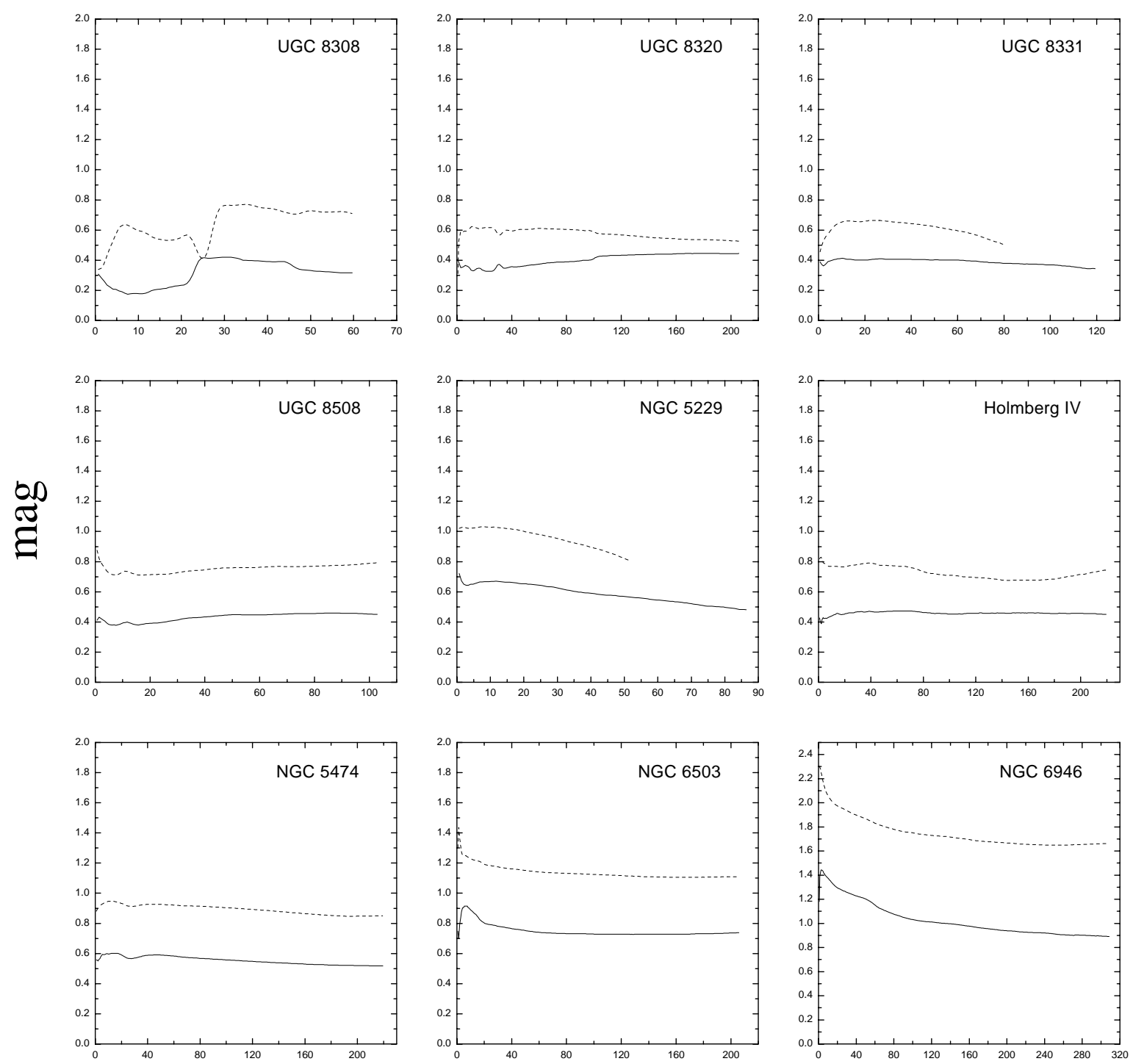

\section{Radius (arcsec)}

Fig. 2. continued

results. For these six objects total magnitude and colour estimations were made only in one large circular aperture.

Note that most observed galaxies show a slow increase in the total $B-V$ colour value from the center to the outer parts of the galaxy. Some spiral galaxies show the reverse colour gradient. For the most galaxies the $V-I$ total colours follow $B-V$ variations.

$(V-I)_{0}$ vs. $(B-V)_{0}$ (the total colours corrected for galactic absorption) are plotted in Fig. 3 for the measured galaxies (filled squares). For comparison, the integrated colours from the papers by Reshetnikov \& Combes (1996) (circles) and Heraudeau \& Simien (1996) (crosses) are also given in the figure. The galaxies from all the three works are well fitted by the following distribution:
$(B-V)_{0}=0.85 \times(V-I)_{0}-0.20$. This line is also plotted in Fig. 3.

Obtaining of azimuthally averaged surface brightness profiles was the next step of the reduction of the observed galaxies. These profiles have an advantage for dwarf galaxies of very low surface brightness and irregular structure (Karachentseva et al. 1996; Papaderos et al. 1996; Bremnes et al. 1998), because they allow the accuracy of the photometry in such galaxies to be improved. Azimuthally averaged surface brightness profiles for our galaxies were obtained by differentiating the galaxy growth curves with respect to radius (Bremnes et al. 1998). The resulting profiles are shown in Fig. 4. Most of the galaxies are calculated till the level of $27-$ $28 \mathrm{mag} / \mathrm{sq} \mathrm{s}$ in $B$ band. Mean errors of the measurements 
Table 4. Total magnitudes of the observed galaxies

\begin{tabular}{|c|c|c|c|c|c|c|c|c|c|c|c|c|}
\hline Ident. & $B_{\mathrm{T}}$ & $V_{\mathrm{T}}$ & $I_{\mathrm{T}}$ & $(B-V)_{\mathrm{T}}$ & $(V-I)_{\mathrm{T}}$ & $R_{25}(B)$ & $B_{25}$ & $R_{25}(V)$ & $V_{25}$ & $R_{25}(I)$ & $I_{25}$ & $M_{B}$ \\
\hline UGC 3755 & 14.07 & 13.52 & 12.64 & 0.55 & 0.88 & 42.5 & 14.26 & 46.0 & 13.68 & 54.2 & 12.74 & -14.39 \\
\hline NGC 2366 & 11.68 & 11.26 & 10.74 & 0.42 & 0.53 & 123.5 & 12.18 & 150.9 & 11.54 & 180.4 & 10.88 & -16.17 \\
\hline UGC 3974 & 13.62 & 13.14 & 12.54 & 0.48 & 0.60 & 41.8 & 15.13 & 57.6 & 14.25 & 67.9 & 13.53 & -14.69 \\
\hline K 52 & 16.35 & 16.11 & 15.55 & 0.24 & 0.56 & & & & & & & -11.11 \\
\hline UGC 4459 & 14.55 & 14.14 & 13.49 & 0.45 & 0.65 & 33.6 & 15.35 & 41.8 & 14.66 & 52.1 & 13.87 & -13.05 \\
\hline UGC 4483 & 14.95 & 14.51 & 14.02 & 0.44 & 0.49 & & & & & & & -12.99 \\
\hline F464-v3 & 16.90 & 16.63 & 16.00 & 0.27 & 0.63 & & & & & & & -11.81 \\
\hline UGC 5086 & 16.45: & 15.89: & 15.17: & 0.56 : & $0.72:$ & & & & & & & $-13.05:$ \\
\hline Holmberg I & 13.64 & 13.22 & 12.68 & 0.42 & 0.54 & 41.8 & 15.51 & 71.3 & 14.11 & 89.2 & 13.21 & -15.66 \\
\hline UGC 5288 & 14.62 & 14.06 & 13.22 & 0.56 & 0.84 & 30.2 & 14.90 & 38.4 & 14.21 & 44.6 & 13.30 & -14.71 \\
\hline BK3N & 18.78 & 18.27 & 17.08 & 0.51 & 1.19 & & & & & & & -8.83 \\
\hline Holmberg IX & 14.53 & 14.31 & 13.49 & 0.22 & 0.82 & 29.5 & 15.95 & 32.2 & 15.61 & 56.9 & 14.30 & -13.48 \\
\hline UGC 5423 & 14.42 & 13.90 & 13.48 & 0.52 & 0.42 & 33.6 & 14.68 & 38.4 & 14.08 & 53.5 & 13.53 & -14.55 \\
\hline UGC 5692 & 13.57 & 12.66 & 11.36 & 0.90 & 1.30 & 53.5 & 14.10 & 80.3 & 12.95 & 122.1 & 11.46 & -14.89 \\
\hline UGC 5918 & 15.00 & 14.58 & 13.78 & 0.42 & 0.80 & 21.3 & 16.62 & 30.9 & 15.65 & 56.9 & 14.16 & -14.41 \\
\hline K 73 & 17.09 & 16.66 & 15.69 & 0.43 & 0.97 & & & & & & & -11.03 \\
\hline UGC 6817 & 13.70 & 13.35 & 12.30 & 0.35 & 1.04 & 52.1 & 14.49 & 63.1 & 13.90 & 106.3 & 12.58 & -14.38 \\
\hline NGC 4068 & 13.19 & 12.77 & 12.01 & 0.42 & 0.76 & 65.2 & 13.44 & 73.4 & 12.95 & 87.8 & 12.09 & -15.49 \\
\hline NGC 4144 & 12.16 & 11.63 & 10.74 & 0.53 & 0.89 & 90.6 & 12.48 & 118.0 & 11.81 & 154.4 & 10.80 & -17.86 \\
\hline NGC 4163 & 13.63 & 13.07 & 12.22 & 0.56 & 0.85 & 46.0 & 13.80 & 56.3 & 13.18 & 67.2 & 12.26 & -14.21 \\
\hline PGC 38998 & 15.37 & 14.82 & 14.10 & 0.55 & 0.73 & 21.3 & 15.53 & 24.7 & 14.95 & 29.5 & 14.13 & -15.73 \\
\hline UGC 7298 & 15.95 & 15.66 & 15.17 & 0.29 & 0.48 & 19.9 & 16.29 & 21.3 & 15.95 & 24.7 & 15.30 & -13.83 \\
\hline UGC 7321 & 13.90 & 13.29 & 12.41 & 0.61 & 0.88 & 40.5 & 14.84 & 58.3 & 13.85 & 83.7 & 12.59 & -14.65 \\
\hline IC 3308 & 15.41 & 14.96 & 13.94 & 0.45 & 1.02 & 20.6 & 15.80 & 22.6 & 15.33 & 36.4 & 14.31 & -12.64 \\
\hline UGC 7577 & 12.95 & 12.36 & 11.36 & 0.59 & 1.00 & 76.1 & 13.41 & 94.7 & 12.69 & 142.7 & 11.51 & -15.55 \\
\hline NGC 4449 & 10.06 & 9.65 & 8.85 & 0.42 & 0.79 & 164.6 & 10.10 & 187.3 & 9.66 & 222.0 & 8.80 & -18.28 \\
\hline UGC 7698 & 13.53 & 13.18 & 12.49 & 0.35 & 0.69 & 55.6 & 14.71 & 65.9 & 14.02 & 91.9 & 12.98 & -15.46 \\
\hline IC 3687 & 13.79 & 13.37 & 12.77 & 0.42 & 0.60 & 50.1 & 14.28 & 57.6 & 13.73 & 69.3 & 12.98 & -13.68 \\
\hline IC 4182 & 12.02 & 11.60 & 10.72 & 0.42 & 0.88 & 123.5 & 12.31 & 140.6 & 11.78 & 173.6 & 10.73 & -16.56 \\
\hline UGC 8201 & 13.31 & 12.97 & 12.27 & 0.34 & 0.70 & 66.5 & 13.69 & 77.5 & 13.24 & 101.5 & 12.39 & -15.24 \\
\hline UGC 8308 & 15.45 & 15.10 & 14.34 & 0.39 & 0.70 & 26.8 & 15.85 & 28.1 & 15.39 & 36.4 & 14.46 & -12.45 \\
\hline UGC 8320 & 12.97 & 12.53 & 11.99 & 0.44 & 0.54 & 72.0 & 13.40 & 85.8 & 12.88 & 100.2 & 12.18 & -15.10 \\
\hline UGC 8331 & 14.46 & 14.17 & 13.62 & 0.29 & 0.55 & 35.7 & 15.03 & 41.8 & 14.49 & 46.6 & 13.79 & -15.15 \\
\hline UGC 8508 & 14.12 & 13.67 & 12.88 & 0.45 & 0.79 & 41.8 & 14.42 & 52.1 & 13.83 & 61.7 & 12.99 & -13.77 \\
\hline NGC 5229 & 14.33 & 13.85 & 13.05 & 0.48 & 0.80 & 39.1 & 14.68 & 43.9 & 14.02 & 43.9 & 13.15 & -14.28 \\
\hline Holmberg IV & 13.76 & 13.31 & 12.57 & 0.45 & 0.74 & 46.0 & 14.51 & 61.1 & 13.79 & 78.9 & 12.84 & -15.90 \\
\hline NGC 5474 & 11.60 & 11.08 & 10.23 & 0.52 & 0.85 & 110.4 & 11.78 & 125.5 & 11.18 & 142.7 & 10.27 & -17.73 \\
\hline NGC 6503 & 11.05 & 10.31 & 9.20 & 0.73 & 1.10 & 116.6 & 11.14 & 140.6 & 10.37 & 198.9 & 9.21 & -17.68 \\
\hline NGC 6946 & 10.00 & 9.11 & 7.46 & 0.89 & 1.65 & 264.8 & 10.06 & 269.6 & 9.15 & 475.0 & 6.80 & -20.38 \\
\hline
\end{tabular}

were estimated by intercomparison of individual profiles for the same objects obtained from different frames in the same passband and they are of about $0.05^{\mathrm{m}}$ at the $22 \mathrm{mag} / \mathrm{sq} \mathrm{s}$ isophotal level and about $0.3-0.4$ at the $27 \mathrm{mag} / \mathrm{sq} \mathrm{s}$ isophotal level in each photometric band.

\subsection{Photometric errors}

Summing up all internal errors given above, we obtain the resulting errors of the total magnitude estimation to be about $0.18^{\mathrm{m}}$ in $B$ band, $0.18^{\mathrm{m}}$ in $V$ band and $0.19^{\mathrm{m}}$ in $I$ band and the resulting errors of surface brightness estimation to be about $0.17^{\mathrm{m}}$ at the $22 \mathrm{mag} / \mathrm{sq} \mathrm{s}$ isophotal level and about $0.35-0.45$ at the $27 \mathrm{mag} / \mathrm{sq} \mathrm{s}$ isophotal level in each photometric band.

External photometric errors can be estimated by a comparison of the total magnitudes of the observed galaxies with the published magnitudes of these galaxies. The comparison of the total $B$-magnitudes estimated in the present work with those given in the article of Bremnes et al. (1998) for seven galaxies in common, yields an agreement of $0.18 \pm 0.04$. A considerable systematic deviation may be caused by the uncertainty 


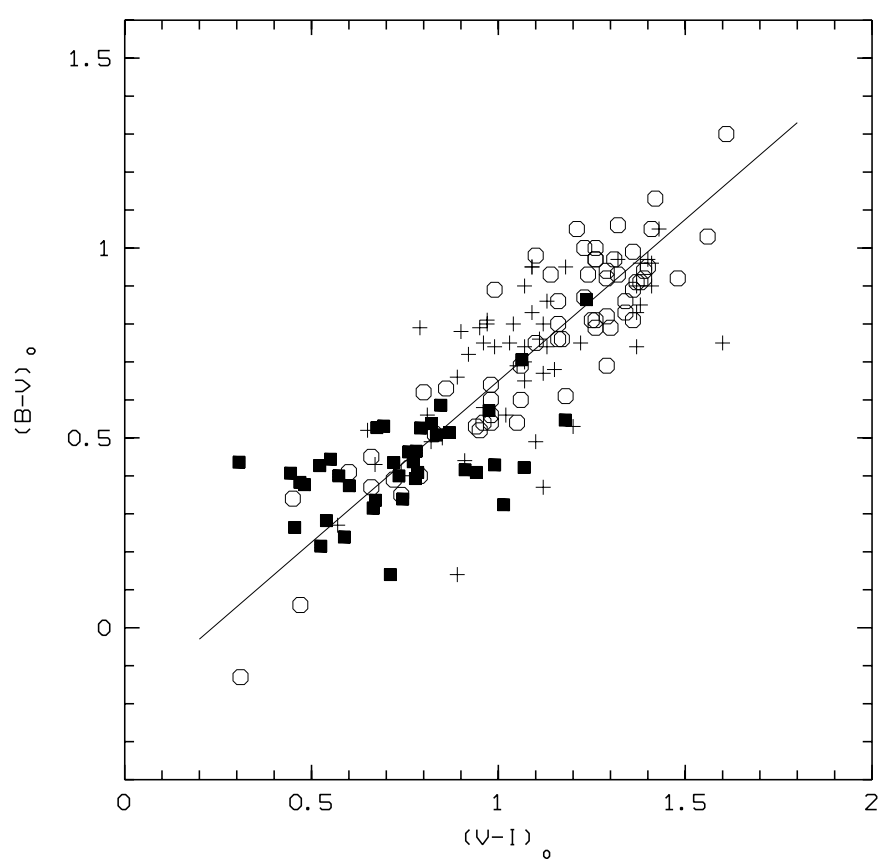

Fig. 3. $(B-V)_{0}$ versus $(V-I)_{0}$ for our sample galaxies (filled squares) and for the galaxies from the article of Heraudeau \& Simien (1996) (crosses) and Reshetnikov \& Combes (1996) (circles). The line of the regression for all galaxies is presented

in the photometric calibration in the work of Bremnes et al., where they give information about the problems due to atmospheric extinction. A second similar work of the same authors has recently become available. A comparison of our data with those obtained by Bremnes et al. (1999), for seven common galaxies shows a good agreement of $0.07 \pm 0.16$.

Total magnitude estimates for some galaxies of our sample can be found in a number of papers. A comparison of these magnitudes with our data is given in Table 5, where the columns are:

\section{Column 1: galaxy name.}

Columns 2, 3 and 4: total magnitude difference in $B, V$ and $I$ band respectively.

Column 5: total $B-V$ colour difference.

Column 6: reference to the work, where magnitudes for the comparison were taken. Here

1: work of Tikhonov \& Karachentsev (1998), where galaxies were observed at the 6-m telescope of Special Astrophysical Observatory, Russia, and CCD field contained only $120^{\prime \prime} \times 80^{\prime \prime}$.

2: article of Makarova \& Karachentsev (1998).

3: article of Makarova et al. (1998) (in [2] and [3] the observations were carried out at the Nordic Optical Telescope with the CCD of $3^{\prime} \times 3^{\prime}$ field).

4: paper of Gavazzi, Boselli (1996).

5: article of Patterson \& Thuan (1996) (note that, unlike for other works, there is no good agreement of our data with the magnitudes given in this paper).
Table 5. Comparison with magnitudes of other authors (see text)

\begin{tabular}{lrrrrr}
\hline Ident. & $\Delta B$ & $\Delta V$ & $\Delta I$ & $\Delta(B-V)$ & Reference \\
\hline K 52 & 0.63 & & 2.61 & & {$[5]$} \\
UGC 4459 & -0.17 & & & & {$[6]$} \\
UGC 4483 & -0.39 & & & & {$[6]$} \\
UGC 5086 & & 0.66 & 1.08 & & {$[2]$} \\
Holmberg I & -0.13 & & & & {$[6]$} \\
BK3N & -0.18 & & & & {$[6]$} \\
UGC 5423 & -1.00 & -0.86 & & {$[5]$} \\
UGC 5692 & -0.01 & & & & {$[6]$} \\
UGC 5918 & -0.21 & & & & {$[6]$} \\
K 73 & -0.17 & & & 0.11 & {$[6]$} \\
NGC 4163 & -0.30 & & & & {$[1]$} \\
UGC 7321 & 0.05 & 0.21 & & 0.14 & {$[1]$} \\
UGC 7577 & & & & 0.00 & {$[3]$} \\
UGC 7698 & 0.38 & & 0.04 & {$[3]$} \\
IC 3687 & 0.04 & & & {$[7]$} \\
UGC 8308 & -0.05 & & & {$[7]$} \\
UGC 8320 & -0.08 & & & {$[7]$} \\
UGC 8331 & 0.19 & & & {$[7]$} \\
UGC 8508 & 0.24 & & & {$[7]$} \\
NGC 5229 & 0.23 & & & {$[7]$} \\
Holmberg IV & 0.11 & & & {$[7]$} \\
NGC 5474 & -0.17 & &
\end{tabular}

6: work of Bremnes et al. (1998).

7: work of Bremnes et al. (1999).

The comparison of our blue total magnitudes and $B-V$ colours with those given in the PGC yields a poor agreement: $0.63 \pm 0.69 \mathrm{mag}$. The large scatter shows that the PGC magnitudes are considerably inhomogeneous. It is possible that the PGC total magnitudes used in photometric investigations may not provide good reliability.

The comparison directly of the aperture magnitudes in each photometric band, which are available for 26 of our galaxies in Prugniel \& Heraudeau (1998), yields a good agreement: $0.00 \pm 0.15$ in $B, 0.04 \pm 0.14$ in $V, 0.09 \pm$ 0.16 in $I$. Mean difference for each galaxy was found for the apertures (at least three) available. The results were then averaged for the all galaxies.

\section{Fitting of surface brightness profiles}

It is well known that surface brightness profiles of dwarf galaxies (both irregular and spheroidal) and also disks of spiral galaxies can be fitted by an exponential intensity law of brightness distribution (de Vaucouleurs 1959)

$I(r)=I_{0} * \exp (-r / h)$

or, in magnitudes per sq $\mathrm{s}$

$\mu(r)=\mu_{0}+1.086 *(r / h)$,

where $\mu_{0}$ is the central surface brightness and $\mathrm{h}$ is the exponential scale length. Most of the measured galaxies are 

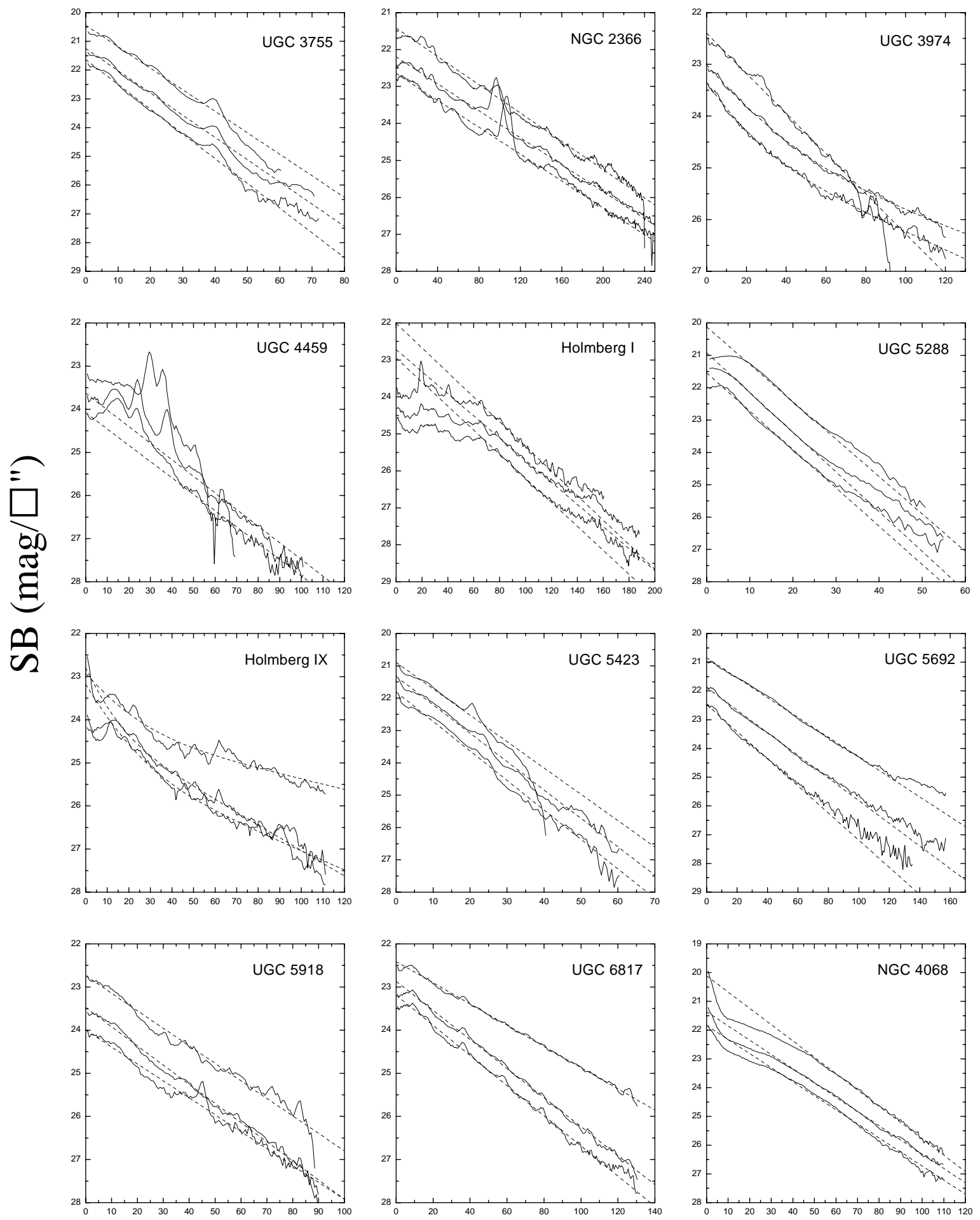

\section{Radius (arcsec)}

Fig. 4. The azimuthally averaged surface brightness profiles for the measured galaxies in $B$ band (bottom lines), those in $V$ band (middle lines) and those in $I$ band (upper lines). The model profiles are shown by the dashed lines 

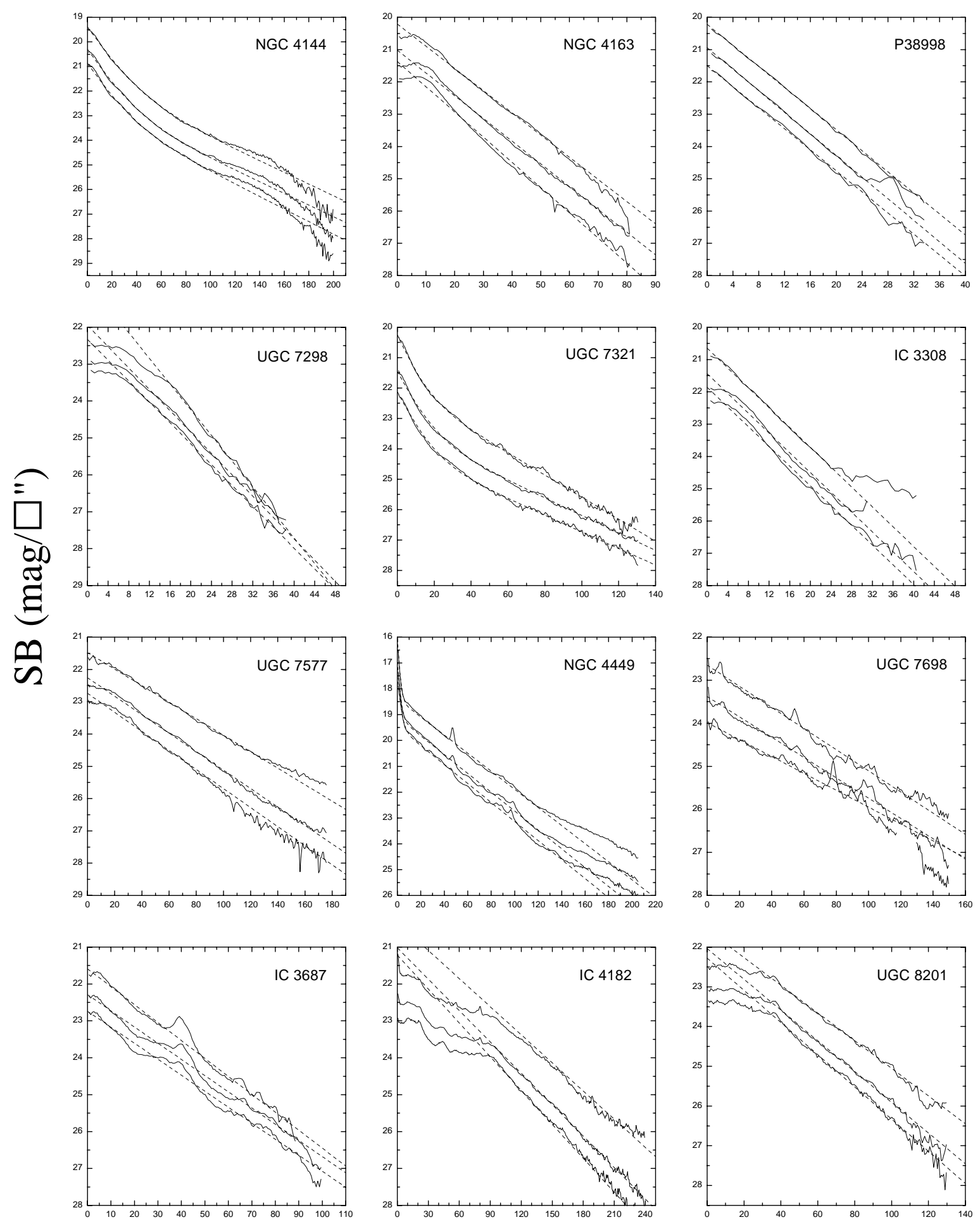

\section{Radius (arcsec)}

Fig. 4. continued 

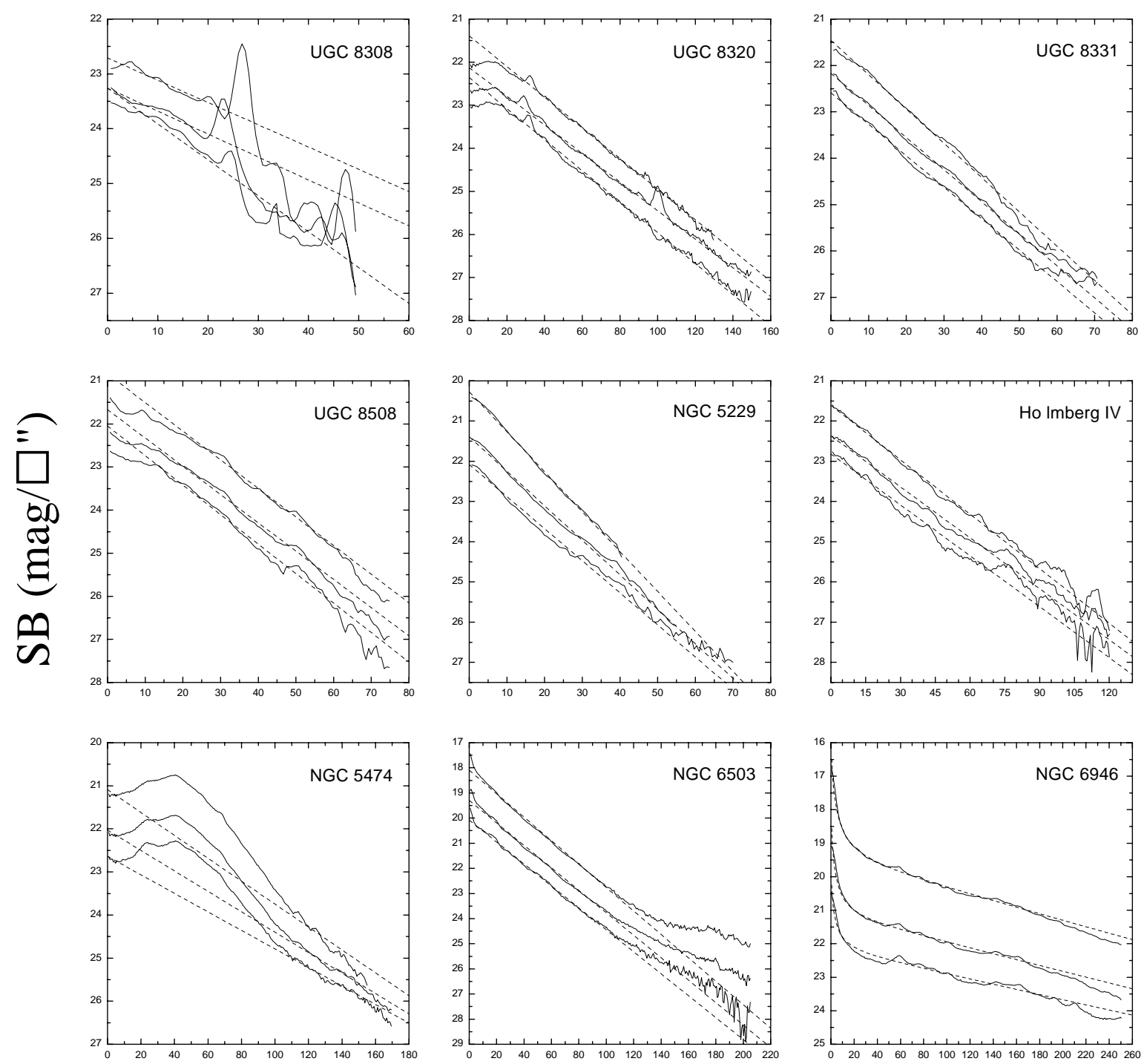

\section{Radius (arcsec)}

Fig. 4. continued

well-fitted by an exponential law in the whole profile or in its outer parts. Some galaxy profiles show a prominent surface brightness central peak, which may be formed by the star formation regions near the center of the galaxy. The best result for these cases is given by decomposition of the profiles into contributions from the two exponential components.

Profile parameters estimation for UGC 4459 in $I$ band has not been made because there is not any significant part of the profile which followed to exponential law of brightness distribution.

Finally, one galaxy of our sample, namely the late type spiral NGC 6946, is well decomposed into bulge and exponential disk components. Among the most commonly used bulge fitting functions is the " $r^{1 / 4}$ law" proposed by de Vaucouleurs (1948). This is written as

$I(r) / I_{\mathrm{e}}=10^{-3.33 *\left(\left(r / r_{\mathrm{e}}\right)^{1 / 4}-1\right)}$

or, in magnitudes per sq $\mathrm{s}$

$\mu(r)=\mu_{\mathrm{e}}+8.33 *\left(\left(r / r_{\mathrm{e}}\right)^{1 / 4}-1\right)$,

where $r_{\mathrm{e}}$ is the effective radius that contains half of the total luminosity, and $\mu_{\mathrm{e}}$ is the effective surface brightness at this radius.

The exponential fits to the surface brightness profiles were done by a least-squares fitting procedure. Decomposition of profiles into two components was performed using the algorithm by Kormendy (1977). 
Table 6. Derived photometric parameters

\begin{tabular}{|c|c|c|c|c|c|c|c|c|c|c|c|c|}
\hline Ident. & $\mu_{0}^{B}$ & $\begin{array}{l}h_{B} \\
(")\end{array}$ & $\mu_{0}^{B 1}$ & $\begin{array}{c}h_{B 1} \\
\text { (") }\end{array}$ & $\mu_{0}^{V}$ & $\begin{array}{l}h_{V} \\
(")\end{array}$ & $\mu_{0}^{V 1}$ & $\begin{array}{c}h_{V 1} \\
\text { (") }\end{array}$ & $\mu_{0}^{I}$ & $\begin{array}{l}h_{I} \\
(")\end{array}$ & $\mu_{0}^{I 1}$ & $\begin{array}{l}h_{I 1} \\
(")\end{array}$ \\
\hline UGC 3755 & 21.64 & 12.62 & & & 21.25 & 14.04 & & & 20.44 & 14.51 & & \\
\hline NGC 2366 & 22.65 & 59.75 & & & 22.20 & 59.61 & & & 21.42 & 56.72 & & \\
\hline UGC 3974 & 24.52 & 62.91 & 23.81 & 15.22 & 24.52 & 79.36 & 23.32 & 20.61 & 22.40 & 28.03 & & \\
\hline UGC 4459 & 24.08 & 28.69 & & & 23.63 & 28.18 & & & & & & \\
\hline Holmberg I & 22.95 & 33.43 & & & 22.72 & 36.31 & & & 22.01 & 32.65 & & \\
\hline UGC 5288 & 21.54 & 9.16 & & & 20.92 & 8.84 & & & 20.12 & 9.39 & & \\
\hline Holmberg IX & 24.85 & 49.45 & 23.45 & 11.35 & 24.08 & 36.75 & 23.20 & 8.14 & 24.18 & 90.66 & 23.34 & 14.46 \\
\hline UGC 5423 & 21.81 & 11.91 & & & 21.33 & 12.37 & & & 20.90 & 13.33 & & \\
\hline UGC 5692 & 22.47 & 23.00 & & & 21.88 & 27.58 & & & 20.85 & 31.45 & & \\
\hline UGC 5918 & 24.00 & 27.66 & & & 23.48 & 24.45 & & & 22.74 & 26.74 & & \\
\hline UGC 6817 & 23.19 & 31.06 & & & 22.87 & 32.25 & & & 22.41 & 44.14 & & \\
\hline NGC 4068 & 21.84 & 22.22 & & & 21.36 & 21.91 & & & 20.11 & 19.15 & & \\
\hline NGC 4144 & 22.72 & 42.60 & 21.08 & 13.42 & 22.38 & 45.95 & 20.47 & 13.80 & 21.52 & 45.88 & 19.56 & 14.07 \\
\hline NGC 4163 & 21.37 & 13.91 & & & 21.02 & 15.42 & & & 20.21 & 15.80 & & \\
\hline P38998 & 21.50 & 6.67 & & & 20.94 & 6.50 & & & 20.22 & 6.66 & & \\
\hline UGC 7298 & 22.33 & 7.68 & & & 21.95 & 7.51 & & & 20.76 & 6.25 & & \\
\hline UGC 7321 & 24.01 & 39.85 & 22.33 & 8.14 & 23.32 & 37.78 & 21.67 & 8.35 & 21.96 & 29.90 & 20.50 & 6.99 \\
\hline IC 3308 & 21.86 & 7.11 & & & 21.44 & 7.09 & & & 20.65 & 7.07 & & \\
\hline UGC 7577 & 22.74 & 36.82 & & & 22.26 & 38.00 & & & 21.48 & 42.48 & & \\
\hline NGC 4449 & 19.46 & 29.51 & 17.72 & 2.05 & 19.09 & 29.79 & 17.12 & 1.66 & 18.39 & 30.99 & 16.21 & 1.41 \\
\hline UGC 7698 & 23.99 & 55.42 & & & 23.38 & 46.01 & & & 22.65 & 44.21 & & \\
\hline IC 3687 & 22.74 & 25.05 & & & 22.29 & 24.74 & & & 21.59 & 22.35 & & \\
\hline IC 4182 & 21.22 & 35.51 & & & 21.02 & 38.31 & & & 20.33 & 42.76 & & \\
\hline UGC 8201 & 22.28 & 26.82 & & & 22.04 & 28.09 & & & 21.57 & 31.19 & & \\
\hline UGC 8308 & 23.26 & 16.59 & & & 23.26 & 25.94 & & & 22.71 & 26.75 & & \\
\hline UGC 8320 & 22.36 & 30.17 & & & 22.13 & 32.72 & & & 21.39 & 30.51 & & \\
\hline UGC 8331 & 22.59 & 16.02 & & & 22.17 & 15.70 & & & 21.46 & 14.69 & & \\
\hline UGC 8508 & 22.05 & 15.86 & & & 21.67 & 16.54 & & & 20.84 & 16.35 & & \\
\hline NGC 5229 & 22.08 & 13.61 & & & 21.39 & 12.65 & & & 20.27 & 10.93 & & \\
\hline Holmberg IV & 22.82 & 25.74 & & & 22.37 & 25.72 & & & 21.61 & 24.06 & & \\
\hline NGC 5474 & 22.64 & 50.53 & & & 22.03 & 45.81 & & & 21.09 & 40.86 & & \\
\hline NGC 6503 & 20.05 & 24.32 & & & 19.35 & 22.57 & & & 18.19 & 22.16 & & \\
\hline NGC 6946 & 22.15 & 142.08 & 26.10 & 63.19 & 21.10 & 125.55 & 24.00 & 41.00 & 19.41 & 113.12 & 22.18 & 59.82 \\
\hline
\end{tabular}

The rms deviation of the derived model profiles from the original ones does not exceed $0.02 \mathrm{mag}$.

The results of the fitting are presented in Table 6 . The columns of the table are:

Column 1: galaxy name.

Columns 2 and 3: best-fitting parameters of the exponential disk in $B$ filter (the central surface brightness $\mu_{0}$ (mag/sq s) and exponential scale length $h(\operatorname{arcsec}))$.

Columns 4 and 5: best-fitting parameters of the second exponential disk in $B$ filter, or, for NGC 6946 are bestfitting bulge parameters (effective surface brightness $\mu_{\mathrm{e}}(\mathrm{mag} / \mathrm{sq} \mathrm{s})$ and effective radius $\left.r_{\mathrm{e}}(\operatorname{arcsec})\right)$.

Columns 6, 7, 8 and 9: same as 2, 3, 4 and 5, but in $V$ filter.

Columns 10, 11, 12 and 13: same as 2, 3, 4 and 5, but in $I$ filter.

The central surface brightnesses in the table are not corrected for galactic absorption.
In Fig. 5 the absolute $B$-magnitudes for the measured galaxies are shown versus the model central surface brightnesses $\left(\mu_{0}^{\mathrm{c}}\right)$ in $B$ band (left) and scale lengths in $\mathrm{kpc}$ (right). All the magnitudes are corrected for galactic absorption. For comparison, these distributions for spiral galaxies studied by Binggeli \& Cameron (1993) and van der Kruit (1987), and also for dwarf and low surface brightness galaxies (LSB) from Vennik et al. (1996) are given in the figure. As seen from Fig. 5 the absolute $B$-magnitudes of the studied galaxies span over a wide range from $-12^{\mathrm{m}}$ to $-20.5^{\mathrm{m}}$ and the exponential disk central surface brightnesses are distributed mostly in the range from 21 to $24.5 \mathrm{mag} / \mathrm{sq} \mathrm{s}$, except some bright spiral galaxies, the central surface brightnesses of which are higher. The mean value of the central SB in $B$ band is about $22.4 \pm 0.8$ for the measured galaxies (and the mean value in $V$ band is about $22.1 \pm 0.9$ and in $I$ band is about $21.3 \pm 0.9$ ). Near the same area is occupied in the figure by the nearby dwarf 

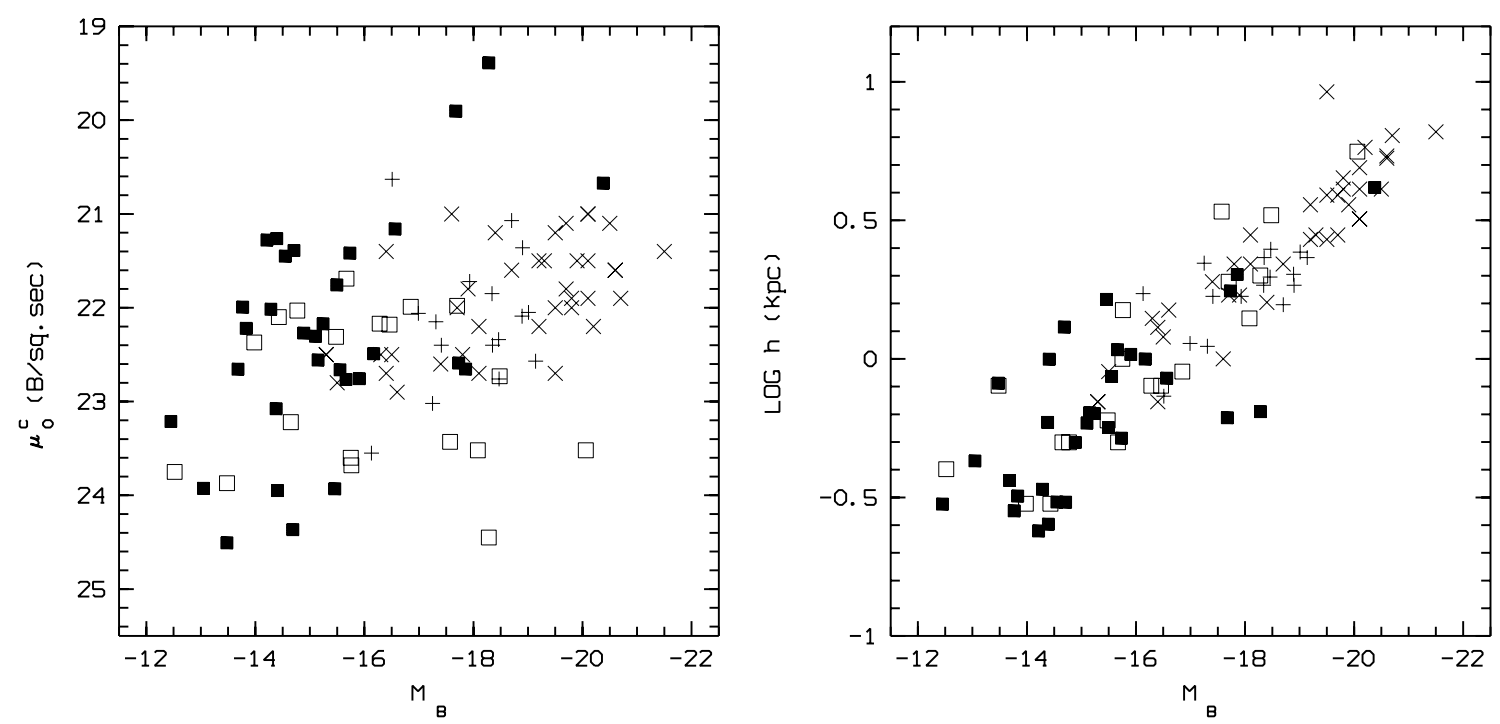

Fig. 5. The model central surface brightnesses (left) and logarithm of the scale lengths (right) in $B$ band versus the absolute $B$-magnitudes. The filled squares are the measured galaxies, the squares are the galaxies from the article of Vennik et al. (1996), the crosses $(+)$ are the spiral galaxies from the work of Binggeli \& Cameron (1993), and the crosses $(\times)$ are the spiral galaxies from the work of van der Kruit (1987)

galaxies studied in the paper by Vennik et al. (1996). Some distant LSB galaxies from this work have higher absolute $B$-magnitudes and lower central surface brightnesses. The plotted spiral galaxies are on average of higher absolute magnitudes and also of higher central SB. Note that absolute magnitudes are only slightly correlated with central surface brightnesses for the galaxies plotted in the Fig. 5. On the contrary, the disk scale lengths in $B$ band are closely correlated with absolute $B$-magnitudes. The scale lengths are distributed over a wide range, $-0.7<\log h<+0.7$ and the area of nearby dwarf galaxies and the area of bright spiral (and LSB) galaxies are well-separated in the figure. The mean scale length value for the measured nearby dwarf galaxies is about $0.68 \pm 0.40 \mathrm{kpc}$ in $B$ band.

\section{Notes to individual galaxies}

NGC 2366. The galaxy has an irregular, clumpy structure. The clump in the lower right part of the galaxy has a separate number in the PGC catalogue, LEDA 93088. However, it may also be a star formation region belonging to NGC 2366. Both of the objects are measured together in the present work.

UGC 4459. A light from a bright foreground star appears at the upper left corner of the image (see Fig. 1).

UGC 4483. The estimates of the total magnitudes for this galaxy contain large uncertainties due to a bright nearby foreground star.

UGC 5086. The galaxy measurements have poor calibration because of atmospheric extinction.

BK 3N. The M 81 boundary appears at the upper left corner of the image (see Fig. 1).
Holmberg IX. According to our measurements, this is one of the bluest galaxies of the sample with $B-V=$ 0.22 . However, it is possible that the result is affected by the bright neighbor $\mathrm{M} 81$.

UGC 7321. This is an edge-on galaxy of big diameter. For the case of this object the method of obtaining an azimuthally averaged profile can probably yield satisfactory results only in the central parts of the galaxy.

NGC 5229. This is also an edge-on object of big diameter. The same notes as for UGC 7321 can be given.

NGC 5474. This bright spiral galaxy has a very asymmetric structure. The geometric center of the galaxy was used when measuring total magnitudes and surface brightness profiles.

NGC 6946. The CCD field is probably too small for this galaxy. Therefore, the given total magnitudes should be taken as lower limits.

\section{Conclusion}

Observations of 39 nearby, mostly dwarf galaxies are presented in this work. The observations were carried out at the 1.2-m telescope of Observatoire de Haute-Provence (France) with $B, V$ and $I$ Cousins filters. The sample of nearby galaxies with significant uncertainties in the total magnitude $B_{\mathrm{T}}$ was selected from the Principal Galaxy Catalogue.

Based on surface and integrated photometry of the observed galaxies we derived total $B, V$ and $I$ magnitudes and integrated $B-V, V-I$ colours and also radii and magnitudes at the $25^{\mathrm{m}}$ isophotal level.

It should be noted that $B-V, V-I$ colours of the measured galaxies (corrected for galactic absorption) 
are well fitted by the following distribution: $(B-V)_{0}=$ $0.85 \times(V-I)_{0}-0.20$.

Azimuthally averaged surface brightness profiles were derived for 33 galaxies in each photometric band. Most of the measured galaxies can be well fitted by an exponential intensity law of brightness distribution in the whole profile or in its outer parts. The best-fitting exponential parameters are given for the galaxies.

The relations between absolute $B$-magnitudes and model central surface brightnesses in $B$ band and scale lengths in $B$ band are also presented. For comparison, similar data are given for spiral galaxies, nearby dwarf galaxies and low surface brightness galaxies from articles of different authors. Our sample galaxies span over a wide range of absolute $B$-magnitudes from $-12^{\mathrm{m}}$ to $-20.5^{\mathrm{m}}$ and the exponential disk central surface brightnesses are distributed mostly in the range from 21 to $24.5 \mathrm{mag} / \mathrm{sq} \mathrm{s}$, except some bright spiral galaxies. Note that absolute magnitudes are weakly correlated with central surface brightnesses for the given data. The mean value of the central SB in $B$ band is about $22.4 \pm 0.8$ for the measured galaxies (and the mean value in $V$ band is about $22.1 \pm 0.9$ and in $I$ band is about $21.3 \pm 0.9$ ). The scale lengths in $B$ band are closely correlated with absolute $B$-magnitudes, and the area of nearby dwarf galaxies and the area of bright spiral (and LSB) galaxies are well-separated in the figure. The mean scale length value for the measured nearby dwarf galaxies is about $0.68 \pm 0.40 \mathrm{kpc}$ in $B$ band.

Acknowledgements. I would like to thank I.D. Karachentsev for help in the sample selection and for consideration given to my work. I am also grateful to D.I. Makarov for useful discussions and also to G.G. Korotkova for her participation in data processing. I would like to thank also P. Prugniel, referee, for his valuable comments. This work is partially supported by INTAS grant No. 1390.

\section{References}

Binggeli B., Cameron L., 1993, A\&AS 98, 297

Bremnes T., Binggeli B., Prugniel P., 1998, A\&AS 129, 313

Bremnes T., Binggeli B., Prugniel P., 1999, A\&AS 137, 337

De Vaucouleurs G., 1948, Ann. d'Astrophys. 11, 247

De Vaucouleurs G., 1959, Handbuch der Physik 53, 275, Flugge S. (ed.). Springer, Berlin

Gavazzi G., Boselli A., 1996, Ap. Let. Comm. 35, 1

Greggio L., Tosi M., Clampin M., De Marchi G., Leitherer C., Nota A., Sirianni M., 1998, ApJ 504, 725

Heraudeau P., Simien F., 1996, A\&AS 118, 111

Karachentsev I., 1994, Astron. Astrophys. Trans. 6, 1

Karachentsev I., Tikhonov N., Sazonova L., 1994, A\&AS 106, 555

Karachentseva V., Prugniel P., Vennik J., Richter G., Thuan T., Martin J., 1996, A\&AS 117, 343

Kormendy J., 1977, ApJ 217, 406

Landolt A., 1992, AJ 104, 340

Makarova L., Karachentsev I., 1998, A\&AS 133, 181

Makarova L., Karachentsev I., Takalo L., Heinamaki P., Valtonen M., 1998, A\&AS 128, 459

Papaderos P., Loose H.-H., Thuan T., Fricke K., 1996, A\&AS 120,207

Patterson R., Thuan T., 1996, Ap\&SS 107, 103

Paturel G., Bottinelli L., Di Nella H., Durand N., Garnier R., Gouguenheim L., Marthinet M., Petit C., Rousseau J., Theureau G., Vauglin I., 1996, Principal Galaxy Catalogue: PGC-ROM 1996, Observatoire de Lyon

Prugniel P., Heraudeau P., 1998, A\&AS 128, 299

Reshetnikov V., Combes F., 1996, A\&AS 116, 417

Schlegel D., Finkbeiner D., Davis M., 1998, ApJ 500, 525

Stetson P., Hesser J., Smecker-Hane T., 1998, PASP 110, 533

Tikhonov N., Karachentsev I., 1998, A\&AS 128, 325

van der Kruit P., 1987, A\&A 173, 59

Vennik J., Richter G., Thanert W., Biering C., 1996, Astron. Nachr. 317, 289 